\title{
How human runners regulate footsteps on uneven terrain
}

\author{
Nihav Dhawale ${ }^{1,2}$ and Madhusudhan Venkadesan ${ }^{1}$ \\ ${ }^{1}$ Department of Mechanical Engineering \& Materials Science, Yale University, USA \\ ${ }^{2}$ National Centre for Biological Sciences, Tata Institute of Fundamental Research, India
}

\begin{abstract}
Running was crucial for human evolution, especially for persistence hunting. But how people remain stable on uneven natural terrain is not understood. In particular, we do not know how runners manage competing demands on their attention, like seeking level ground areas to step on versus seeking out prey. Previous work used step-like terrain with height variations but not the more destabilizing combination of height and slope variations like a natural undulating terrain. So we studied human runners on undulating uneven terrain and measured their energetics, kinematics, and ground forces. Here we show that runners do not selectively land their feet on more level areas. Instead, they remain stable using their body's mechanical response and possibly occasional feedback corrections, with little increase in energy consumption relative to flat ground. These findings may explain how persistence hunters can devote visual attention to tasks other than stability while running on uneven ground.
\end{abstract}

Running on uneven terrain was needed for persistence hunting and played a key role in human evolution $[1,2]$. Visual guidance of foot placement may help avoid potentially destabilizing areas of the ground $[3,4]$. But vision is also needed to track subtle footprints, avoid large obstacles, and continuously survey the landscape for prey. So there are potentially competing demands on visual attention. Indeed, studies of people walking on uneven ground show subtle interactions between gaze, stepping patterns, and terrain complexity that trade-off the use of vision to guide the next step versus planning the overall path through uneven terrain $[5,6]$. This trade-off is probably more severe in running because there is lesser time to visually gather information about the terrain for the oncoming step and use that to plan the step. Furthermore, in persistence hunting, the runner should balance these demands on stability and attention against running economy and metabolic energy consumption [7]. We currently do not know how people negotiate stability on natural terrain and its impact on metabolic energy consumption.

Past studies of uneven terrain running have investigated running over step-like terrain comprised of piecewise flat blocks on the ground [8-12]. Such terrain are not representative of the undulating unevenness of natural environments, but show how humans deal with height variations on the ground. A key finding is that there is only a slight increase of around $5 \%$ in metabolic energy consumption to run on these step-like, piecewise flat terrain [9]. That 
naturally raises the question of how the runners remain stable.

Stability analysis has relied on interpreting data using models that approximate the runner as a point-like mass on a massless leg, the widely used spring-legged inverted pendulum (SLIP) model $[8,11,13-15]$. The main hypothesized strategies to aid stability include higher leg retraction rates [16], wider lateral foot placement [9, 17], and the possible use of vision to guide foot placement [18]. Point-mass models are appropriate to interpret stability for step-like terrain because slope variations do not influence stability in point-mass models [19]. The point-mass has no moment of inertia about its center, because of which the ground force is constrained to point towards the center of mass, and the ground slope becomes immaterial. Theoretical work using models that have a non-zero moment of inertia show that slope variations are far more destabilizing [19], consistent with common experience among runners. So there is a need for experiments on undulating terrain with slope variations to understand how natural terrain affects stability.

Mathematical analysis of running finds two key strategies for remaining stable when both the slope and height of the terrain randomly vary [19]. The first strategy is to selectively step on flatter regions like local maxima and minima of an undulating terrain where the ground is always level. Thus, the undulating terrain is effectively just height variations, and the previously proposed stabilization mechanisms on step-like terrain become effective. The second strategy is related to the mathematical result that step-to-step fluctuations in impulses arising from tangential interactions of the foot with uneven ground are most destabilizing and could cause the runner to fall. Thus controlling the body's mechanical state at touchdown to reduce the horizontal impulse will aid stability. Although sensory feedback control is necessary for stability according to the model, the combined effect of controlling the footstep location and reducing the horizontal impulse allows for feedback acting slowly over multiple steps to suffice. However, experimental tests of these hypothetical foot placement and horizontal impulse modulation strategies are currently lacking.

The cumulative understanding of running from past experiments on flat and step-like terrain and models on uneven terrain motivates this paper's two hypotheses. The first hypothesis predicts that runners will land their feet on level ground areas to reduce destabilization by randomly varying slopes. The second hypothesis predicts that runners will reduce tangential impulses with the ground during stance. However, feedback control will probably be important over several steps and often manifests as increased variability in step-to-step kinematic variables 

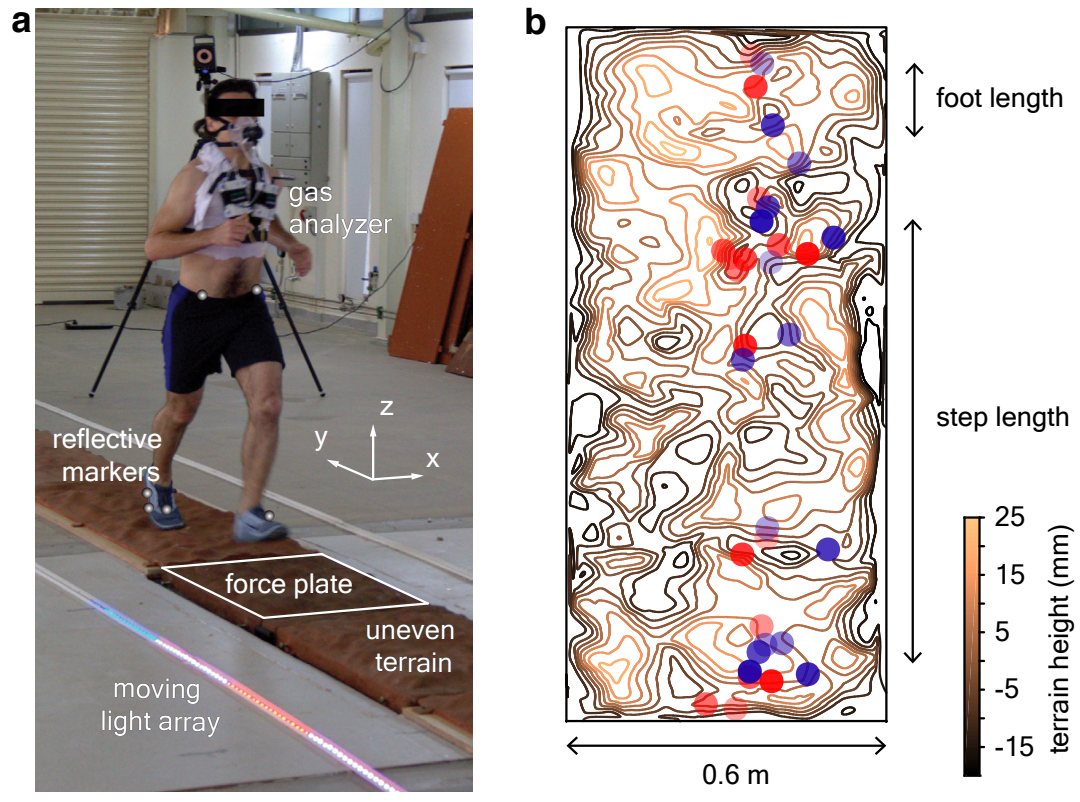

Fig. 1. Uneven terrain experiments. a, We conducted human-subject experiments on flat and uneven terrain while recording biomechanical and metabolic data. The reflective markers and the outline of the force plate are digitally exaggerated for clarity. b, Footsteps were recorded to determine whether terrain geometry influences stepping location, illustrated here by a meansubtracted contour plot of terrain height (units of $\mathrm{mm}$ ) for an approximately 6 foot segment of uneven II overlaid with footsteps. Blue and red circles represent opposite directions of travel and marker transparency differentiates trials.

such as step length and width as found by previous studies $[4,9,15]$. So we studied human runners on uneven terrain that had slight but potentially destabilizing undulations and measured their energetics, kinematics, and foot contact forces (Fig. 1).

\section{Results}

We conducted overground running experiments with nine subjects who ran on three surfacesflat, uneven $I$, and uneven $I I$-while measuring ground reaction forces, oxygen consumption, and three-dimensional kinematics of the subject's hip and feet (Fig. 1a, Methods). We probed foot placement strategies by digitizing the uneven terrain and using those data in a stochastic analysis of the foot kinematics (Fig. 1b, 2). In addition, we estimated the tangential impulse that causes loss of forward momentum in early stance using the measured ground reaction forces (Fig. 3a,b), and recorded step kinematics and energetics that allow comparison with previously published studies on uneven terrain locomotion [9, 20-22].

Statistical tests used linear mixed models with a significance threshold of $\alpha=0.05$. Post-hoc, pairwise comparisons with Tukey corrections were performed for the ANOVAs and ANCOVAs that passed the significance threshold (methods 1.4.2). We report statistically significant com- 

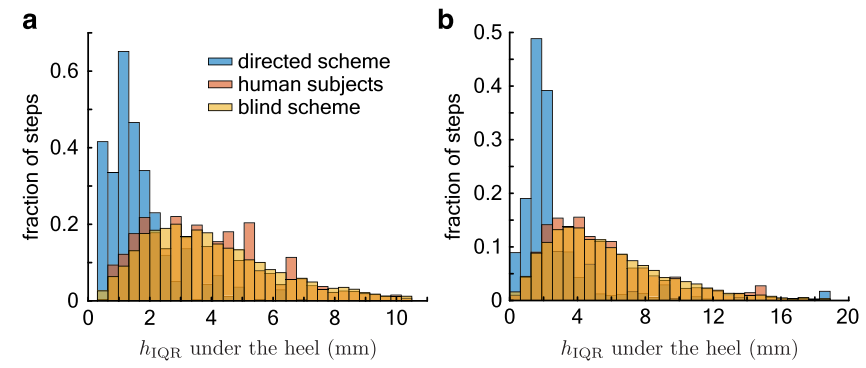

Fig. 2. Foot placement on uneven terrain. Histogram of the interquartile range of heights $\left(h_{I Q R}\right)$ at footstep locations for the directed sampling scheme (blue), experiments (red, 5237 footsteps), and the blind sampling scheme (yellow) on $\mathbf{a}$, uneven I and $\mathbf{b}$, uneven II. Note that $h_{\mathrm{IQR}}$ varies over a greater range on uneven II.

parisons in the text and all comparisons in supplementary Tables S1,S2. Differences in the mean between testing conditions are reported as mean \pm standard error (S.E.) of the mean. Descriptive statistics are reported as mean \pm standard deviation (S.D.).

Nondimensionalization: We express lengths in units of leg length $\ell$ and speed in units of $\sqrt{g \ell}$, [23] where $g$ is acceleration due to gravity. Statistically significant post-hoc comparisons are additionally reported in dimensional units using $g=9.81 \mathrm{~m} / \mathrm{s}^{2}$, and the mean of the measurements across subjects, namely, $\ell=0.89 \mathrm{~m}$ and $m=66.1 \mathrm{~kg}$.

\section{Foot placement on uneven terrain}

To test whether real runners prefer to land on flatter patches, the measured footsteps were compared against two extreme models, a null hypothesis of a blind runner and an alternative hypothesis of a directed runner whose footsteps are selectively aimed at level parts of the terrain.

All participants were heel-strike runners on all terrain types as judged from the double peak in the vertical ground reaction force profile. So their footsteps sample the terrain in heel-sized patches. Therefore, we quantized the terrain into heel-size patches and used the interquartile range of heights $\left(h_{\mathrm{IQR}}\right)$ in each patch as a measure of its unevenness.

The blind scheme uses a uniform random sample of heel-sized patches of the terrain to obtain statistics of the terrain at landing locations. The directed scheme preferentially samples more level patches using a Markov chain Monte Carlo (MCMC) model (methods 1.2.1). In this model, each step is directed towards a patch of the lowest $h_{\mathrm{IQR}}$, but within bounds defined by the mean and standard deviation of the experimentally measured step length and width.

The experimentally measured stepping patterns are indistinguishable from the blind scheme on both uneven I and II in terms of the terrain unevenness as quantified by $h_{\mathrm{IQR}}$ (human subjects versus blind scheme in Fig. 2). However, the directed scheme finds substantially more 
level landing patches, showing that it was possible for the runners to land on more level ground (directed scheme in Fig. 2). These trends are also borne out in a subject-wise analysis (supplementary Fig. S2,S3).

The directed scheme found more level patches at the cost of increased variability in step length and step width compared to the runners. The mean step length of the directed scheme was $0.3 \%$ and $0.02 \%$ lower than experimental data on uneven I and uneven II, respectively. However, the standard deviation of step length increased by $22 \%$ on uneven I and by $18 \%$ on uneven II compared to experimental measurements. The mean step width under the directed scheme was slightly different from the experimental data; higher by $3.7 \%$ on uneven I and lower by $7 \%$ on uneven II. In contrast, the standard deviation of step width for the directed scheme was higher than the experimental measurements by $97 \%$ on uneven I and by $79 \%$ on uneven II.

The overall statistics of the terrain location at foot landing may obscure step-to-step dependence of the foot landing on terrain features. So we used a second analysis of footstep patterns that estimated the foot landing probability $p_{i, j}$ in the $(i, j)^{\text {th }}$ foot-sized cell and correlated that with terrain unevenness as defined by the median and the interquartile range of the terrain height in that cell. Positive correlation with the median height would indicate stepping on local maxima that are flatter than the surrounding, and negative correlation with the interquartile range would indicate stepping on flatter regions with more uniform height. Neither correlation was significant (Table 1). Subject-wise landing probabilities also bear out the same results (supplementary fig S5). Taken together, these results indicate that the runners did not guide their footsteps towards flatter areas of the terrain.

Table 1. Correlation between landing probability and terrain unevenness. Details of the ANCOVAs on the linear mixed models from equation (12) showing denominator degrees of freedom, F-values, and p-values from the dataset of stepping probabilities and terrain height statistics of 1628 foot-sized terrain patches for all subjects on uneven I and uneven II.

\begin{tabular}{llll}
\hline dependent variable & DenDF & F-value & p-value \\
\hline median terrain height & 11.8 & 0.40 & 0.54 \\
IQR terrain height & 26.4 & 0.02 & 0.89 \\
\hline
\end{tabular}

\section{Fore-aft impulses}

The fore-aft ground reaction force in stance initially decelerates the center of mass before accelerating it forward (Fig. 3a). We find that less than $6 \pm 1 \%$ (mean \pm S.D.) of the forward momentum is lost during the deceleration phase of stance and there is no dependence on ter- 
bioRxiv preprint doi: https://doi.org/10.1101/2021.02.22.432238; this version posted February $22,2021$. The copyright holder for this preprint (which was not certified by peer review) is the author/funder, who has granted bioRxiv a license to display the preprint in perpetuity. It is made available under aCC-BY 4.0 International license.

rain or subject (Fig. 3b). The low variability of the fore-aft impulse, just $1 \%$ of the forward momentum, suggests that it is tightly regulated across runners, terrain and steps.

a

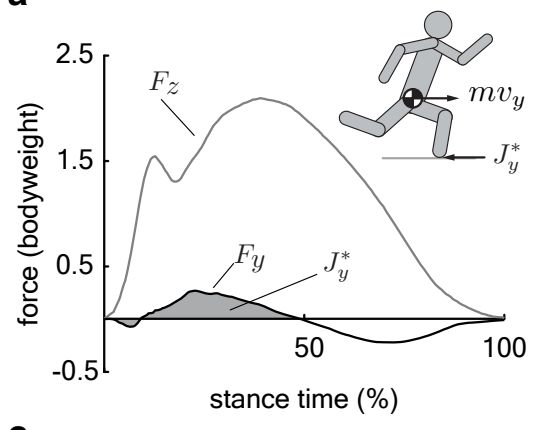

C

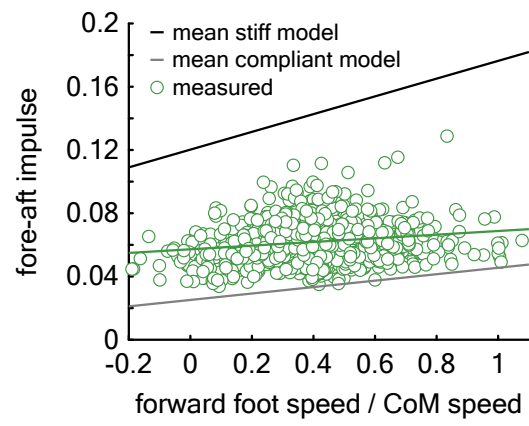

b

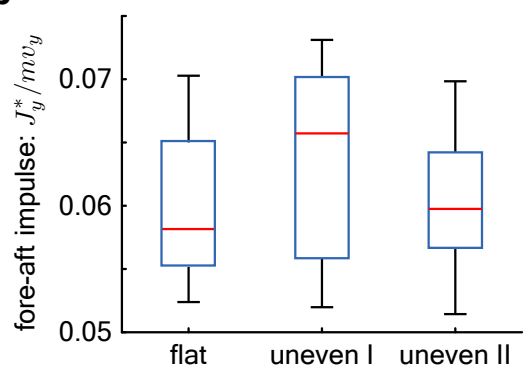

d

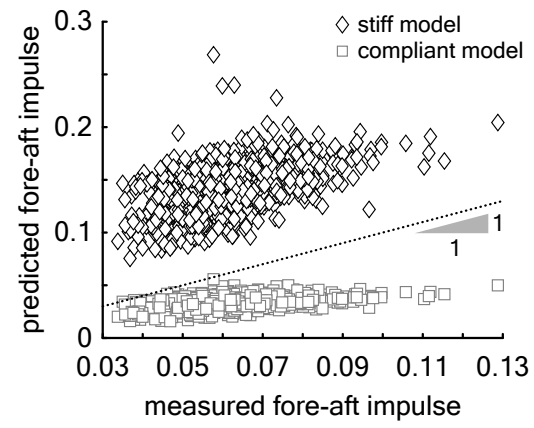

Fig. 3. Regulation of fore-aft impulses. a, The fore-aft impulse $J_{y}^{*}$ (grey shaded area) is found by integrating the measured fore-aft ground reaction force $F_{y}$ (black curve) during the deceleration phase. b, Mean $\frac{J_{y}^{*}}{m v_{y}}$. c, Measured $\frac{J_{y}^{*}}{m v_{y}}$ (green circles) versus relative forward foot speed at landing (forward foot speed/center of mass speed) for each step recorded on all terrain types (total 1081 steps). The green line is the regression fit for the data. The dark and light grey lines are the predicted fore-aft impulse for the mean stiff and compliant jointed models, respectively. Per step model predictions in supplementary Fig. S6. d, Measured versus predicted fore-aft impulses for every step. The dotted line represents perfect prediction.

Fore-aft impulses can be reduced by reducing forward foot speed at landing or by maintaining compliant joints at touchdown [19]. The regulation of foot speed is unlikely to be the primary determinant of the low variability in the collision impulse because the dimensionless forward foot speed at touchdown across all terrain varied by nearly $50 \%$ of its mean $(0.4 \pm 0.2$, supplementary Table S1), whereas fore-aft collision impulses varied only by $17 \%$ of the mean. A statistical analysis lends further support and shows that the dimensionless fore-aft impulse depends significantly, but only weakly, on the dimensionless forward foot speed at landing (supplementary Table $\mathrm{S} 2, p=0.001$, slope $=0.01 \pm 0.003)$.

To further investigate this weak dependence of the retarding impulse on foot speed, we analysed the mechanics of foot landing and the resultant impulse using a four-link chain model of the leg and torso when the foot undergoes a rigid, inelastic collision with the ground (meth- 
ods 1.3). The inertia of the leg's segments are represented by the three links and the torso, arms, and head by a single link attached to the top of the leg (Fig. 7, similar to [24]). The measured kinematics are fed through the model under two stiffness regimes; zero joint stiffness and infinite joint stiffness. Recall that only these two stiffness regimes are distinguishable in instantaneous collision models $[24,25]$, thus requiring no fitting parameters. By analyzing the collisional impulse for these two extremes of joint stiffness, we isolate the contributions to the fore-aft impulse arising from varying the joint stiffness versus varying the forward foot speed at landing.

The estimated fore-aft impulse at both joint stiffness extremes depends linearly on the forward foot speed (supplementary Fig. S6). The models at the two extremes of joint stiffness bound the experimental data, with the compliant model underestimating the measured foreaft impulse while the stiff model overestimates it (Fig. 3c,d, supplementary Fig. S6). This is expected because the muscle contraction needed for weight support and propulsion would induce non-zero but non-infinite stiffness at the joints. But surprisingly, both models overestimate the dependence of the fore-aft impulse on foot speed, although the compliant model is closer to the data (Fig. 3c). The slope of measured speed-impulse data is $0.01 \pm 0.003, p=0.001$ (supplementary Table S2) while that of the compliant jointed model is $0.0203 \pm 0.010, p<0.0001$ and the stiff-jointed model is $0.056 \pm 0.005, p<0.0001$ (supplementary Fig. S6). The compliant model's predictions resemble the data when the measured impulse is below 0.07 (Fig. 3d), which is the case for most steps (Fig. 3b). But the model underestimates the impulse on the occasional steps when runners experience more severe fore-aft impulses. Unlike the compliant model, the stiff model consistently over-estimates the measured fore-aft impulse over its entire range. Thus, we propose that maintaining low joint stiffness at landing helps maintain low fore-aft impulses despite variations in touchdown foot speed.

\section{Leg retraction}

Increased leg retraction rate results in reduced forward foot speed at touchdown, thereby altering the tangential impulse $[16,19]$. The mean non-dimensional forward foot speed at landing is terrain-dependent and lower by $0.17 \pm 0.04(p=0.001)$ on uneven I compared to flat ground, and by $0.15 \pm 0.04(p=0.002)$ on uneven II compared to flat ground (Fig. 4a). For the mean subject, these correspond to reductions of $0.48 \pm 0.11 \mathrm{~m} / \mathrm{s}$ on uneven I and $0.42 \pm 0.11 \mathrm{~m} / \mathrm{s}$ on uneven II compared to flat ground. These differences indicate higher leg retraction rates on 

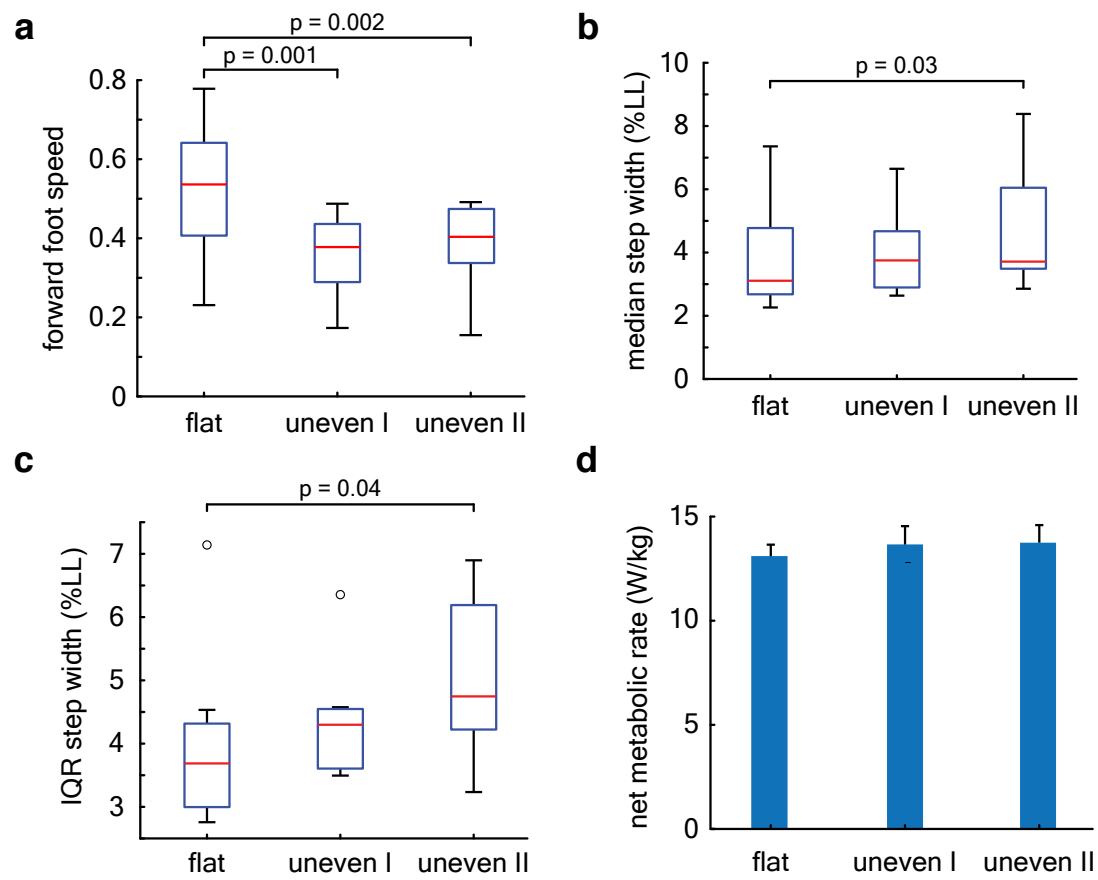

d

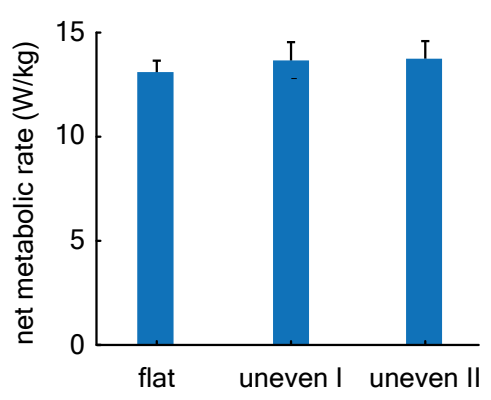

Fig. 4. Energetics and stepping kinematics. a, Box plot of the mean forward foot speed at landing (units of froude number). b, Box plot of the median step width (normalized to leg length). c, Box plot of the step width variability. Central red lines denote the median, boxes represent the interquartile range, whiskers extend to 1.5 times the quartile range, and open circles denote outliers. d, Net metabolic rate normalized to subject mass. Whiskers represent standard deviation across subjects.

uneven terrain, as also reported in running birds $[18,26]$.

Leg retraction has been hypothesized to improve running stability in the context of pointmass models by altering touchdown angle to aid stability [21, 27]. We find that touchdown angle depends significantly but only weakly on forward foot speed at landing ( $p \approx 0$, slope $=0.07 \pm 0.01 \mathrm{rad})$. If the dimensionless forward foot speed at landing varied through its entire observed range from -0.2 to 1.1 , it would result in a change in landing angle of $0.08 \mathrm{rad}$ or $5^{\circ}$.

\section{Stepping kinematics}

Consistent with previous studies on step-like terrain, [9] we find that the median non-dimensional step width is terrain dependent (Fig. 4b) and increased on uneven II versus flat ground by $0.004 \pm 0.001(p=0.03)$. Step width variability, i.e. the interquartile range of step widths within a trial, is also terrain dependent $(p=0.05$, Fig. $4 c)$ and greater on uneven II versus level ground by $0.005 \pm 0.002(p=0.04)$. For the mean subject, median step width increased by $4 \pm 1 \mathrm{~mm}$ and the step width variability (IQR) increased by $6 \pm 2 \mathrm{~mm}$. 


\section{Energetics}

Despite the lack of guided foot placement on level ground and terrain-dependent increases in step width statistics and leg retraction rate, the approximately $5 \%$ increase in metabolic power consumption on the uneven terrain compared to flat we measured was not statistically significant. The statistical significance of the ANOVA, $p=0.08$ (Fig. $4 \mathrm{~d}$ ), was just above our threshold of $\alpha=0.05$. Our findings agree on the estimated increase of around $5 \%$ with running experiments on step-like terrain. [9] However, unlike our statistical results, Voloshina and Ferris [9] report statistical significance $(p<0.05)$.

\section{Discussion}

We find the surprising result that the runners do not use visual information about unevenness to guide their footsteps. Instead, the fore-aft collisions that they experience seem almost decoupled from the horizontal speed with which their foot lands on the ground. Using a model-based analyses of the data, we propose that low joint stiffness was probably the means to maintain low fore-aft impulses, which is known to weaken instabilities associated with the risk of falling by tumbling [19]. Taken together, these results suggest that runners rely on their body's passive mechanical response for remaining stable on uneven terrain. But that does not mean that sensory feedback is not used. We found changes in step width kinematics on the uneven terrain versus flat that were of similar magnitude to previous measurements comparing running on step-like terrain to level ground [9]. These suggest that subjects possibly combined open-loop strategies with step-to-step feedback-mediated stepping strategies that have been previously proposed $[15,28]$.

Fore-aft impulses have not been previously examined in the context of stability. A previous theoretical analysis hypothesized that reducing tangential collisions and maintaining low foreaft impulses reduces the risk of falling by tumbling in the sagittal-plane [19]. Our data are consistent with this model. We find that only $6 \pm 1 \%$ of the forward momentum was lost in stance although the forward foot speed at landing varied by nearly $50 \%$. This reduction in variability is surprising because, all else held the same, speed and impulse are expected to be linearly related. This suggests that the fore-aft impulse is tightly regulated by other means. By examining the role of leg joint compliance using a model-based analysis of the data, we found that the measured fore-aft impulses were partly consistent with an idealized extreme of zero stiffness in the joints at the point of landing. However, joint stiffness in a real runner cannot be 
too small because it is needed to withstand the torques for weight support and propulsion. Thus, we propose that the low variability in fore-aft impulses arises from active regulation of joint stiffness, but a causal relationship between the two remains to be established using methods such as leg EMG measurements [9].

Previous studies based on SLIP models cannot be compared with our results because SLIP models are energy conserving and unaffected by slope variations. However, previous experimental data that have been analyzed using SLIP models to infer leg forces and injury risk point to other benefits of decoupling fore-aft collisions from the foot speed. As we show, the runners appear to not be careful about where precisely they step and could thus encounter occasional sudden drops or step-ups on the terrain. So, being able to vary foot speed and leg retraction rates without affecting stability may allow the runner to maintain consistent leg forces and reduce discomfort or injury risk when sudden or large-obstacles are encountered $[14,26]$. But caution is warranted in comparing our measurements with these past studies because of key differences in the nature of the terrain. As we previously noted, running over natural undulating terrain have not been studied previously and earlier work used step-like terrain. Furthermore, the peak-to-peak height variation of our terrain was less than $6 \%$ of the leg length, but previous studies used much larger step-like obstacles of $10 \%$ leg length or more. For example, we see no change in the variability of the leg landing angle between flat and uneven terrain trials (supplementary Table S1), which is expected if leg landing angle responded to variations in terrain height $[14,26]$. So large step-like obstacles induce different swing-leg control strategies compared with undulating terrain with smaller height variations.

Variability in step-to-step kinematics were largely consistent with previous studies on steplike terrain with some notable differences. Studies of running birds hypothesize that crouched postures could aid stability on uneven terrain [18, 29], as do human-subject data on step-like terrain [9]. We find a slight decrease in the virtual leg length at touchdown on the most uneven terrain compared to flat, but the difference was only around $1 \%$ of the leg length (supplementary Table S1), whose effect on stability would be negligible. Human-subject experiments on step-like terrain report changes in step width and step length variability of $27 \%$ and $26 \%$, respectively, and no changes to mean step length or step width between flat and step-like terrain [9]. Like those studies, we find increases in step width variability of $24 \%$ on uneven terrain compared to flat, and additionally find changes in median step width (13\%) but not in step length variability (Fig. 4b, supplementary Table S1). The increase in median step width that 
we measure could be due to the added stability challenges of running on terrain with slope variations in addition to height variations. Further experiments on terrain with similar height statistics but different slope statistics would be required to test this hypothesis.

Unlike studies on step-like terrain [9], we do not find a significant increase in metabolic power consumption on uneven terrain versus flat ground. Caution is needed in interpreting statistical significance when the p-values are at the cusp of significance. Further studies are needed to explain this variance of results between step-like versus undulating terrain, but it may indicate greater trial-to-trial variance on natural uneven terrain. Nevertheless, the magnitude of the estimated difference in metabolic energy consumption, of around $5 \%$, is quite minor in our study as in previous work.

We find no evidence that subjects used visual information from the terrain geometry to plan footsteps despite predicted advantages to stability [19] and evidence from walking studies that highlight the role of vision in guiding step placement on natural, uneven terrain [5]. We speculate that foot placement strategies are used for obstacle avoidance [30] on more complex terrain while our terrain were designed to be undulating and obstacle-free. Our data thus suggests that terrain-guided foot placement strategies are not required for stability on gently undulating terrain but leaves open the possibility that there is a skill-learning component to such foot placement strategies which we could not measure since our volunteers were not experienced trail runners. Further experiments with runners of varying skill levels would be required to directly test this hypothesis.

The absence of terrain-guided foot placement in our experiment could also be the outcome of the need to reduce metabolic energy consumption. Aiming to land on flatter regions of the terrain would probably increase step length and step width variability as shown by the Markov chain model. These are known to increase metabolic energy consumption [31, 32]. The additional stability gains from the foot placement strategy may be marginal and not commensurate with the unfavorable increase in metabolic energy consumption. In this regime, foot placement could be dominated by step-to-step feedback control for stability as found in level treadmill experiments [15]. Experiments with runners on terrain with a wider range of unevenness properties would be required to investigate this hypothetical trade-off between stability and energy consumption. 


\section{Conclusions}

Footsteps were not directed towards flatter regions of the terrain despite predicted benefits to stability. This suggests that the measured variability in step placement might arise from passive dynamics or step-to-step feedback control, as found in earlier studies on flat and step-like terrain. Instead we find evidence for a previously uncharacterized control strategy, where the destabilizing effects of careless stepping may be partly mitigated by the stabilizing influence of low tangential collisions coupled with the bodys mechanical response. This combination of feedback and body mechanics helps limit the reliance on visual guidance of footsteps. Importantly, the limited need for visual attention may explain how running hunters can track subtle footprints and hunt prey on uneven terrain without falling.

\section{Methods}

\subsection{Protocol and experimental measurements}

We conducted overground running experiments with 9 subjects ( 8 men, 1 woman; age 23-45 years, body mass $66.1 \pm 8.5 \mathrm{~kg}$, leg length $0.89 \pm 0.04 \mathrm{~m}$, reported as mean $\pm \mathrm{SD})$. All subjects were able-bodied, ran approximately $30 \mathrm{~km}$ per week, and had run at least one half-marathon or marathon within the previous year. Experiments were conducted at the National Centre for Biological Sciences, Bangalore, India with informed consent from the volunteers, and IRB approval.

Subjects ran back-and-forth on three $24 \mathrm{~m}$ long and $0.6 \mathrm{~m}$ wide tracks (Fig. 5a). In addition to a flat track, we used two custom-made uneven tracks, uneven I and uneven II, which had increasing unevenness. Uneven I and uneven II had peak-to-valley height differences (amplitude) of $18 \pm 6 \mathrm{~mm}$ and $28 \pm 11 \mathrm{~mm}$ (mean $\pm \mathrm{SD}$ ), respectively, and peak-to-peak horizontal separation (wavelength) of $102 \pm 45 \mathrm{~mm}$ and $108 \pm 52 \mathrm{~mm}$, respectively (Fig. 5b,c,d). We recorded kinematics using an 8-camera motion capture system (Vicon Inc., Oxford, UK) at 300 frames per second and measured the ground reaction forces at $600 \mathrm{~Hz}$ using two force plates (AMTI Inc., model BP600900) embedded beneath the center of the track. Breath-by-breath respirometry was also recorded by a mobile gas analyser (Oxycon Mobile ${ }^{\mathrm{TM}}$, CareFusion Inc.).

A single trial consisted of a 3 minute period of standing when the resting metabolic rate was recorded followed by subjects running back-and-forth on the track for 8-10 minutes. Each subject ran on all three terrain with the order of presentation of the terrain types randomized. 
bioRxiv preprint doi: https://doi.org/10.1101/2021.02.22.432238; this version posted February 22, 2021. The copyright holder for this preprint (which was not certified by peer review) is the author/funder, who has granted bioRxiv a license to display the preprint in perpetuity. It is made available under aCC-BY 4.0 International license.

We controlled the running speed using a moving light array in $24 \mathrm{~m}$ long LED strips laid on either side of the track (Fig. 5a). Subjects were instructed to stay within the bounds of a $3 \mathrm{~m}$ illuminated segment of the LED strip that travelled at $3 \mathrm{~m} / \mathrm{s}$. Running speed fluctuated within a trial, however mean speed as well as speed variability were consistent across terrain types (Table S1). Subjects were provided with standardized, commercially available running shoes.
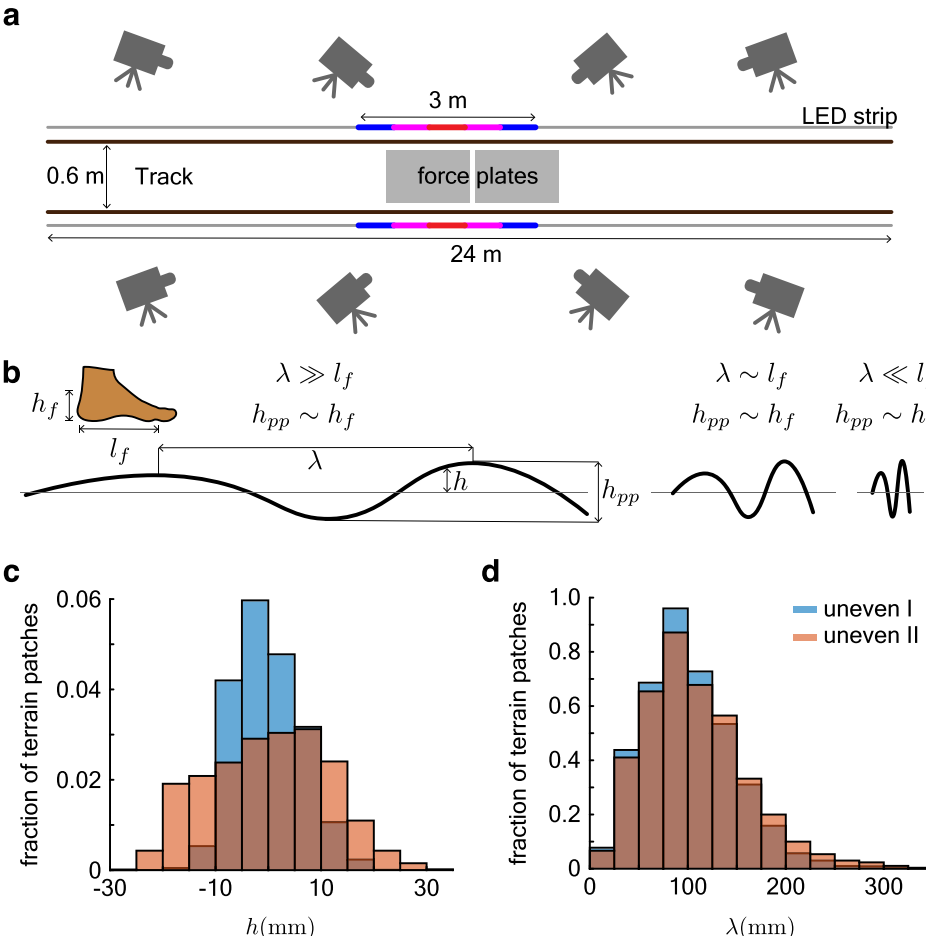

ce plates

$4 \mathrm{~m}$
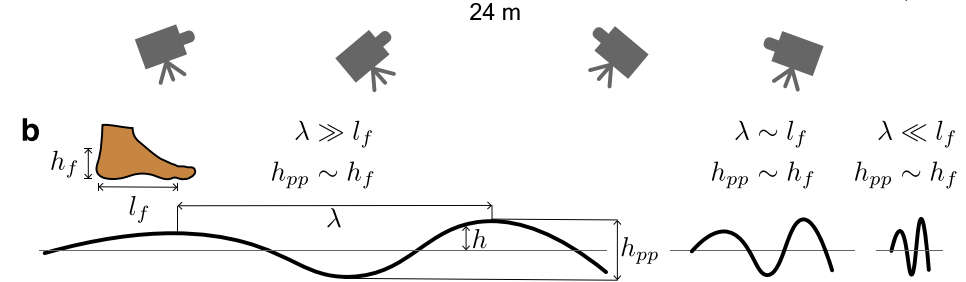

c

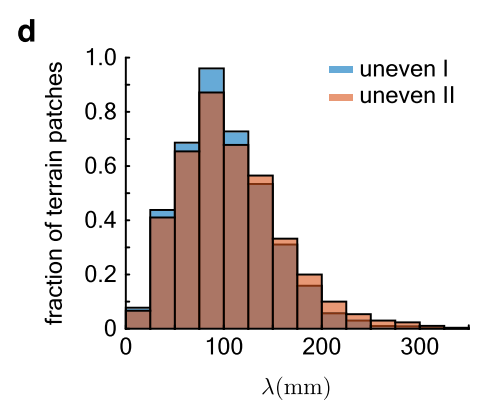

Fig. 5. Details of the experiment design. a, Schematic of the running track, camera placement, force plate positions and the LED strip with a $3 \mathrm{~m}$ illuminated section. $\mathbf{b}$, The terrain was designed so that the range of its height distribution $h$ was comparable to ankle height $h_{\mathrm{pp}} \sim h_{\mathrm{f}}$ and peak-to-peak distances $\lambda$ (along the length of the track) were comparable to foot length $\lambda \sim l_{f}$. c, Histograms of the mean subtracted heights $h$ of the uneven terrain. d, Histograms of the peak-to-peak separation $\lambda$ of the uneven terrain.

\subsubsection{Uneven terrain}

Terrain unevenness was heuristically specified so that peak-to-valley height variations were comparable to ankle height, and peak-to-peak horizontal distances were comparable to foot length (Fig. 5b). Larger terrain height variations with similar (or smaller) peak-to-peak horizontal distances may elicit obstacle avoidance strategies which are not the subject of this paper. Smaller peak-to-valley heights (say, comparable to the height of the heel pad) and smaller peak-to-peak horizontal distances will likely be smoothed out by foot and sole compliance [33], whereas peakto-peak horizontal distances that are longer than a step length may make the slope variation trivial. 
The uneven terrain were constructed by Mars Adventures Inc. (Bangalore, India) by laying fibre glass over heuristically created contours. Epoxy was used to harden the fibre glass sheets into a stiff shell which was coated with a slurry of sand and epoxy to create a surface that texturally resembles weathered rock. The terrain was then digitized using a dense arrangement of reflective markers that were recorded by the motion capture system.

\subsubsection{Kinematics}

Foot kinematics were recorded using fiducial markers that were fixed to the shoes over the calcaneus, second distal metatarsal head, and below the lateral malleolus. Markers were attached to the hip, over the left and right lateral superior iliac spine, and the left and right posterior superior iliac spine. The mean position of the hip markers was used to estimate the center of mass location.

Stance was defined as when the heel marker's forward velocity was minimized and its height was within $15 \mathrm{~mm}$ of the marker's height during standing. The threshold of $15 \mathrm{~mm}$ was chosen to account for terrain height variations so that stance may be detected even when the heel lands on a local peak of the uneven terrain.

The distance $d_{\text {step }}$ covered by the center of mass between consecutive touchdown events and the time $t_{\text {step }}$ between these events define the center of mass speed $v=d_{\text {step }} / t_{\text {step }}$. Leg angle at touchdown was defined as the angle between the vertical and the line formed by joining the heel marker to the center of mass. Virtual leg length at touchdown is defined as the distance between the heel marker and the center of mass. Foot length $l_{f}$ is defined as the average distance in the horizontal plane between the toe and heel marker, across all subjects. Meander away from a straight trajectory is defined as $\left(d-d_{0}\right) / d_{0}$, where $d$ is the distance traversed by the center of mass in the horizontal plane during a single run across the length of the track, while $d_{0}$ is the length of the straight-line fit to the center of mass trajectory. The center of mass trajectory during stance was fitted with a regression line in the horizontal plane and twice the distance of nearest approach of the foot from this line is defined as the step width. This definition allows for the runner's center of mass trajectory to meander while preserving a definition of step width that is consistent with those previously used $[32,34]$. Foot velocity/center of mass velocity at landing were calculated by fitting a cubic polynomial to the heel marker trajectory/center of mass trajectory in a $100 \mathrm{~ms}$ window before touchdown, and calculating the time derivative of the fitted polynomial at the moment just prior to touchdown. Leg retraction rate $\omega$ is determined 
using $\omega=v_{f} /\|\vec{l}\|$, where $v_{f}$ is the component of the foot's relative velocity with respect to the center of mass that is perpendicular to the virtual leg vector $\vec{l}$ (vector joining heel to center of mass).

Step width, step length and virtual leg length at touchdown are normalized by the subject's leg length, defined as the distance between the greater trochanter and lateral malleolus.

\subsubsection{Kinetics}

Force plate data were low-pass filtered using an 8th order, zero-phase, Butterworth filter with a cut-off frequency of $270 \mathrm{~Hz}$. Touchdown on the force plates was defined by a threshold for the vertical force of four standard deviations above the mean unloaded baseline reading.

The forward collision impulse was reported as the decelerating portion of the fore-aft impulse $J_{y}^{*}$ normalized by the aerial phase forward momentum $\left(m v_{y}\right)$, where

$$
J_{y}^{*}=\max _{t}\left|\int_{0}^{t} F_{y}(\tau) d \tau\right|
$$

and $F_{y}$ is the fore-aft component of the ground reaction force.

\subsubsection{Energetics}

Net metabolic rate is defined as the resting metabolic power consumption subtracted from the power consumption during running and normalized by the runner's mass. Metabolic power consumption is determined using measurements of the rate of $\mathrm{O}_{2}$ consumption and $\mathrm{CO}_{2}$ consumption using formulae from Brockway [35]. For running, this is calculated after discarding the first 3 minutes of the run to eliminate the effect of transients. The resting metabolic power consumption is calculated after discarding the first minute of the standing period of the trial. Data from each trial was visually inspected to ensure that the rate of $\mathrm{O}_{2}$ consumption and $\mathrm{CO}_{2}$ production had reached a steady state, seen as a plateau in the data trace.

\subsection{Foot stepping analysis}

\subsubsection{Directed foot placement scheme}

A Markov chain Monte Carlo model finds stepping locations with the lowest terrain unevenness subject to constraints of matching experimentally measured stepping kinematics. Starting from an initial position $\left(x_{i}, y_{i}\right)$, the model finds the next stepping location $\left(x_{i+1}, y_{i+1}\right)$ in the following 
stages: open-loop step, minimization step, and a noise process given by,

$$
\begin{array}{cc}
\text { open-loop step: } & \hat{x}_{i+1}=x_{i}+(-1)^{i} s_{w}, \hat{y}_{i+1}=y_{i}+(-1)^{j} s_{l} . \\
\text { minimization: } & \left(x_{i+1}^{\prime}, y_{i+1}^{\prime}\right)=\arg \min _{(x, y)} t(x, y), \\
x & \in\left[\hat{x}_{i+1}-\sigma_{s w}, \hat{x}_{i+1}+\sigma_{s w}\right], \\
y & \in\left[\hat{y}_{i+1}-\sigma_{s l}, \hat{y}_{i+1}+\sigma_{s l}\right] . \\
\text { noise process: } & x_{i+1}=x_{i+1}^{\prime}+\eta_{x}, y_{i+1}=y_{i+1}^{\prime}+\eta_{y},
\end{array}
$$

where $\eta_{x} \sim v_{M}\left(1,0, \sigma_{s w}\right), \eta_{y} \sim v_{M}\left(1,0, \sigma_{s l}\right)$.

In the open-loop stage, the model takes a step forward and sideways dictated by mean step length $s_{l}$ and mean step width $s_{w}$, respectively. The exponent $j$ is either 0 or 1 and keeps track of the direction of travel. The function $t(x, y)$ evaluates the interquartile range of heights of a heel-sized patch centered around position $(x, y)$. In the minimization step, the model conducts a bounded search about $\left(\hat{x}_{i+1}, \hat{y}_{i+1}\right)$ for the location that minimizes $t(x, y)$. The search region is defined by the standard deviations of the measured step width $\sigma_{s w}$ and step length $\sigma_{s l}$. To simulate sensorimotor noise, the location of this minimum $\left(x_{i+1}^{\prime}, y_{i+1}^{\prime}\right)$ is perturbed by random variables $\eta_{x}, \eta_{y}$. The random variables are drawn from von Mises distributions with $\kappa=1$, centered about zero, and scaled so that the base of support for the distributions are $2 \sigma_{s w}$ and $2 \sigma_{s l}$, respectively.

At the ends of the track, the $x$ position of the runner is reset so that the runner is at the center of the track, and the direction of travel is reversed ( $j$ value is toggled). We simulate for 100,000 steps to ensure that reported terrain statistics at footstep locations as well as step length and step width converge, i.e. errors between simulations in these parameters are less than $1 \%$ of their mean value.

\subsubsection{Quantifying foot placement patterns}

We define a foot placement index to estimate the probability that the runner's foot lands within a foot-sized patch of the track. To calculate this index, we first divide the terrain into a grid of 0.5 foot lengths $\times 1.0$ foot lengths cells, with the longer side of the cell along the length of the track (Fig. 6a). We count the number of footsteps $f_{i, j}$ in each cell $c_{i, j}$, where $i$ indexes the position of the cell along the length of the track and $j$ indexes the position of cell transverse to the track. We also define step length-sized neighborhoods that contain cell $c_{i, j}$ which are one 
a

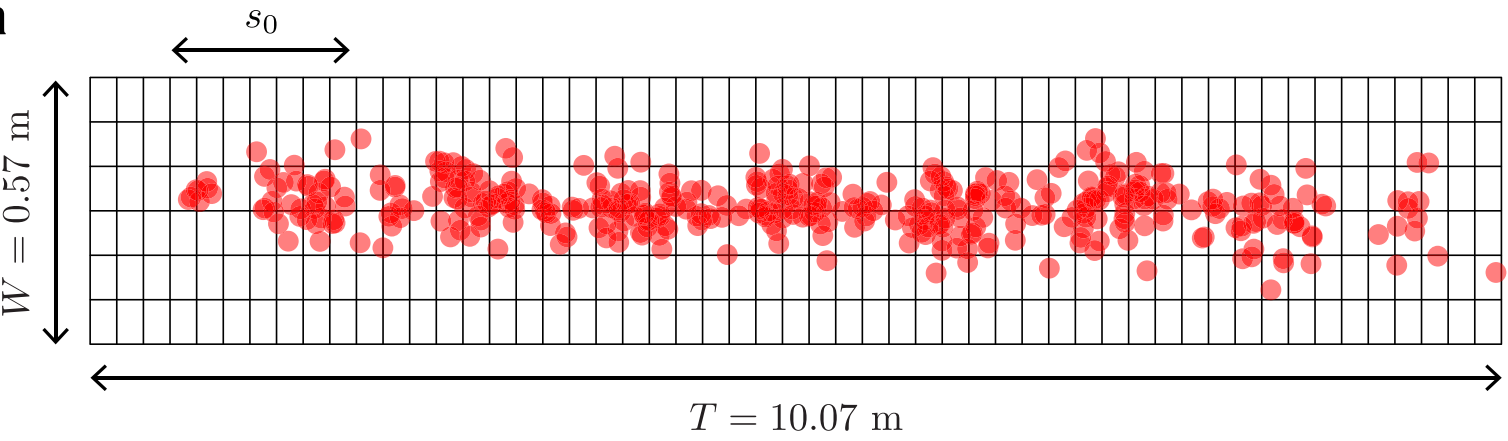

b

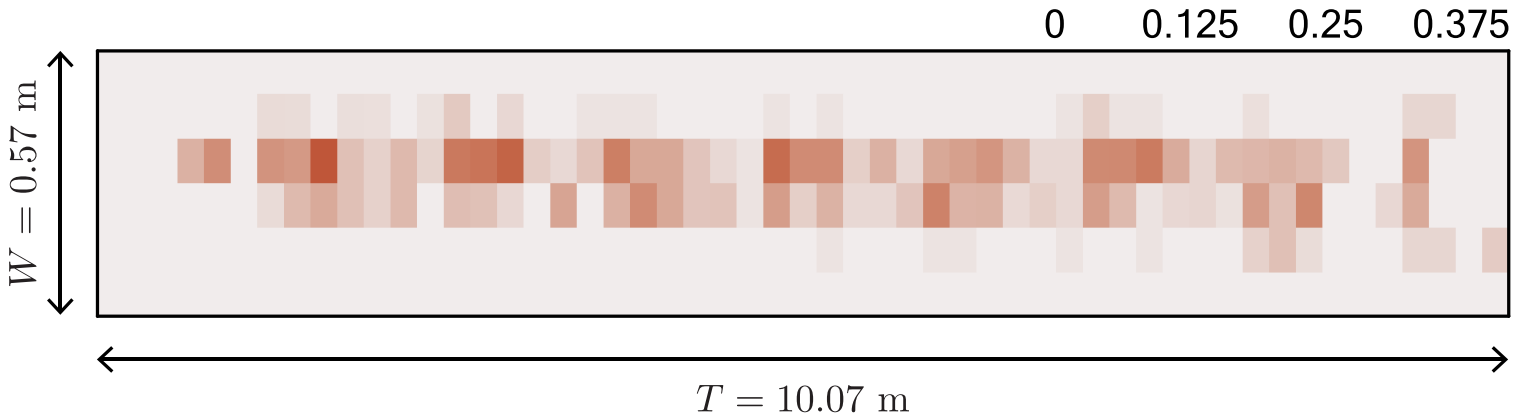

Fig. 6. Foot placement analysis. a, Red circles denote footstep locations (392 footsteps) in the ' $x-y$ ' plane for a representative trial on uneven II. The grid spacing is $190 \mathrm{~mm}$ along the length of the track and $95 \mathrm{~mm}$ along its width. Step length $s_{0}$ is shown for reference. $T$ is the length of the capture volume and $W$ is the width of the track. $\mathbf{b}$, The probability of landing on a foot-sized region of the track is quantified by the foot placement index equation (5) shown as a heatmap with the colour bar at the top left.

step-length long and as wide as the track. Each such neighborhood has a cumulative footstep count $S_{i}$ that depends on the longitudinal location $i$ of the cell. The average across all such step length-sized neighborhoods that contain cell $c_{i, j}$ is $S$. This average $S$ is used to normalize each $f_{i, j}$ to yield the foot placement index $p_{i, j}$ according to,

$$
p_{i, j}=\frac{f_{i, j}}{S} .
$$

The index $p_{i, j}$ measures the fraction of times a foot lands in cell $c_{i, j}$ compared to all other cells that are within a step length distance of it (Fig. 6b). If runners were perfectly periodic with no variation in footstep location from one run over the terrain to the next, $p_{i, j}=1$ for cells on which subjects stepped and $p_{i, j}=0$ otherwise. If, however, stepping location was the result of a uniform random process, $p_{i, j}$ would be a constant for every cell of the terrain.

Heatmaps of the foot placement index $p_{i, j}$ show that runners primarily run down the center of the track with a few preferred stepping locations (hot spots in the heatmap in supplementary Fig. S4). In certain heatmaps, such as subject 06 on uneven I, the runner appears to be 
running diagonally (supplementary Fig. S4). This is due to a misalignment between the axes of the motion capture reference frame and the track. We also report the total number of footsteps recorded for each trial in supplementary Table S3.

To probe foot placement strategies we determine whether the foot placement index $p_{i, j}$ correlates with the median height or the interquartile range of heights within the cell $c_{i, j}$ (supplementary Fig. S5). We test this hypothesis through the use of a statistical model described in section 1.4.2.

\subsection{Collision model}

We model the leg in the sagittal plane as three rods; thigh, foot and shank, connected by hinge joints that are either infinitely compliant or rigid. A fourth rod representing the torso sits on top of the thigh. The rods are assumed to have a uniform mass distribution. Their masses and lengths are expressed as fractions of the measured body mass and leg length using the ratios found by Dempster [36]. The collision is assumed to be instantaneous and inelastic, with a point-contact between the leg and the ground. The instantaneous collision assumption implies that the ground reaction force dominates the collision, thus simplifying the model by ignoring gravitational forces. Models with instantaneous and inelastic collisions are widely used to model stance dynamics for walking $[37,38]$ and running $[19,39]$. We use experimental data on center of mass velocity and leg retraction rate just prior to landing along with the leg angle at touchdown to compute a predicted collisional impulse. Because all our runner's were heel-strikers, we use foot-strike index $s=0.15$ for the collision calculations [24]. The ratio of the collisional impulse to the measured whole body momentum just prior to landing is calculated for the model at the two joint stiffness extremes and compared with experimental measurements of the normalized fore-aft impulse.

Notation: Notation used in this section is as follows. Scalars are denoted by italic symbols (e.g. $I$ for the moment of inertia), vectors by bold, italic symbols ( $\vec{v}$ for velocity), and points or landmarks in capitalized non-italic symbols (such as center of mass G in Fig. 7a). Vectors associated with a point, such as the velocity of center of mass $\mathrm{G}$ are written as $\vec{v}_{\mathrm{G}}$, with the upper-case alphabet in the subscript specifying the point in the plane. Moment of inertia variables are subscripted with '/A' representing the moment of inertia computed about point A, such as $I_{/ G}$ representing the moment of inertia about the center of mass G. Position vectors are denoted by $\vec{r}_{\mathrm{A} / \mathrm{B}}$ which denotes the vector from point $\mathrm{A}$ to point $\mathrm{B}$. Variables just before 
bioRxiv preprint doi: https://doi.org/10.1101/2021.02.22.432238; this version posted February 22, 2021. The copyright holder for this preprint (which was not certified by peer review) is the author/funder, who has granted bioRxiv a license to display the preprint in perpetuity. It is made available under aCC-BY 4.0 International license.

the collision with the terrain are denoted by the superscript '-', and just after the collision by the superscript ' + '. Equations with variables that have no superscript are true at all instances in time.

a

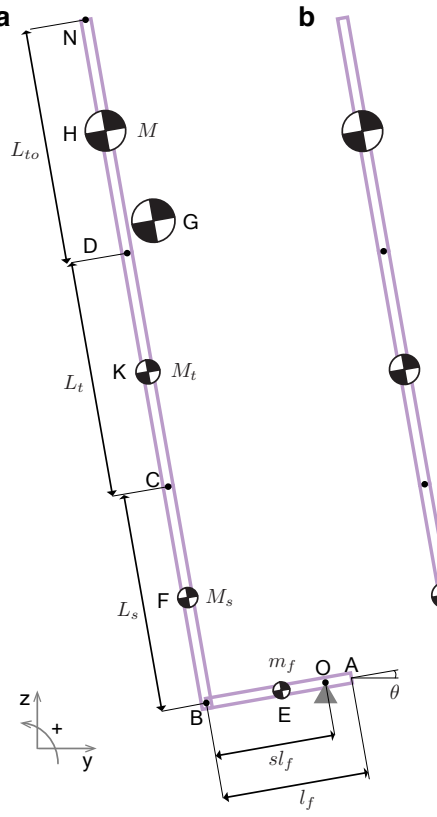

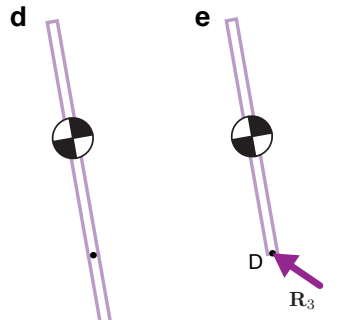

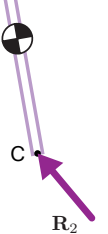

Fig. 7. Model for estimating fore-aft collision impulses from kinematic data. a, A four link model of the foot (A-B), shank (B-C), thigh (C-D), and torso (D-N) moving with center of mass velocity $\vec{v}_{\mathrm{G}}$ and angular velocity $\vec{\Omega}^{-}$collides with the ground at angle $\theta$. G represents the center of mass. Leg length and body mass obtained from data and scaled according to Dempster [36] to obtain segment lengths and masses. Free-body diagrams show all non-zero external impulses: $\mathbf{b}$, collisional impulse $\mathbf{J}$ acting at $\mathrm{O}$, and panels $\mathbf{c}$, d, e, show reaction impulses $\mathbf{R}_{1}, \mathbf{R}_{2}$, and $\mathbf{R}_{3}$ acting at $\mathrm{B}, \mathrm{C}$, and $\mathrm{D}$ respectively.

Rigid Joints: Consider the L-shaped bar (Fig. 7a) falling with velocity $\vec{v}_{\mathrm{G}}^{-}=v_{y}^{-} \hat{\jmath}+v_{z}^{-} \hat{k}$ and rotational velocity $\vec{\Omega}^{-}=\omega^{-} \hat{\imath}$. Upon contact with the ground, the point $\mathrm{O}$ on the foot instantly comes to rest and the center of mass velocity undergoes an abrupt change, $\vec{v}_{\mathrm{G}}^{+}=v_{y}^{+} \hat{\jmath}+v_{z}^{+} \hat{k}, \vec{\Omega}^{+}=$ $\omega^{+} \hat{\imath}$. Due to the instantaneous collision assumption, finite forces like the gravitational force do not contribute to the collisional impulse and hence the ground reaction force acting at point $\mathrm{O}$ contributes the only non-zero external impulse $\vec{J}$ on the body (Fig. $7 \mathrm{~b}$ ). The velocity of the leg and torso after the collision is obtained using angular momentum balance about the contact point and using the kinematic relation for rolling motion about O. These are written as,

$$
\begin{aligned}
M_{b} \vec{r}_{\mathrm{G} / \mathrm{O}} \times \vec{v}_{\mathrm{G}}+I_{/ \mathrm{G}} \vec{\Omega}^{-} & =M_{b} \vec{r}_{\mathrm{G} / \mathrm{O}} \times \vec{v}_{\mathrm{G}}^{+}+I_{/ \mathrm{G}} \vec{\Omega}^{+}, \\
\vec{v}_{\mathrm{G}} & =\vec{v}_{\mathrm{O}}+\vec{\Omega} \times \vec{r}_{\mathrm{G} / \mathrm{O}},
\end{aligned}
$$

$$
\text { where } \vec{v}_{\mathrm{O}}^{+}=0 \text {. }
$$


In the above equations, $M_{b}=M+M_{t}+M_{s}+m_{f}$ is the total mass of the runner. We solve for $\omega^{+}$in equation (6) and obtain the post-collision center of mass velocity $\vec{v}_{\mathrm{G}}^{+}$using equation $6 \mathrm{~b}$. From this, the collision impulse $\vec{J}$ and the normalized fore-aft collisional impulse $\left|J_{y}^{*}\right| / J_{b}$ are calculated using,

$$
\begin{aligned}
\vec{J} & =M_{b}\left(\vec{v}_{\mathrm{G}}^{+}-\vec{v}_{\mathrm{G}}\right), \\
J_{y}^{*} & =\vec{J} \cdot \hat{\jmath}, \\
\text { and } J_{b} & =M_{b}\left(\vec{v}_{\mathrm{G}} \cdot \hat{\vec{\jmath}}\right) .
\end{aligned}
$$

Compliant joints: If the L-bar has compliant joints, then the post-collision velocities for each segment may vary. Therefore, we write additional angular momentum balance equations for each segment to solve for the post-collision state. Since the only non-zero external impulse acting on the shank, thigh, and torso segments is the reaction impulse $\vec{R}_{1}$ acting at B (Fig. 7c), the only non-zero external impulse on the thigh and torso portion of the leg is the reaction impulse $\vec{R}_{2}$ acting at C (Fig. $7 \mathrm{~d}$ ), and the only non-zero external impulse acting on the torso portion of the leg is the reaction impulse $\vec{R}_{3}$ acting at D (Fig. 7e), we write angular momentum balance equations for the entire body and these three segments as,

$$
\begin{aligned}
& M_{b} \vec{r}_{\mathrm{G} / \mathrm{O}} \times v_{\mathrm{G}}^{-}+I_{/ \mathrm{G}} \vec{\Omega}^{-}=m_{f} \vec{r}_{\mathrm{E} / \mathrm{O}} \times \vec{v}_{\mathrm{E}}^{+}+I_{/ \mathrm{E}} \vec{\Omega}_{\mathrm{E}}^{+}+ \\
& M_{s} \vec{r}_{\mathrm{F} / \mathrm{O}} \times \vec{v}_{\mathrm{F}}^{+}+I_{/ \mathrm{F}} \vec{\Omega}_{\mathrm{F}}^{+}+ \\
& M_{t} \vec{r}_{\mathrm{K} / \mathrm{O}} \times \vec{v}_{\mathrm{K}}^{+}+I_{/ \mathrm{K}} \vec{\Omega}_{\mathrm{K}}^{+}+ \\
& M \vec{r}_{\mathrm{H} / \mathrm{O}} \times \vec{v}_{\mathrm{H}}^{+}+I_{/ \mathrm{H}} \vec{\Omega}_{\mathrm{H}}^{+}, \\
& M_{s} \vec{r}_{\mathrm{F} / \mathrm{B}} \times \vec{v}_{\mathrm{F}}+M_{t} \vec{r}_{\mathrm{K} / \mathrm{B}} \times \vec{v}_{\mathrm{K}}+ \\
& M \vec{r}_{\mathrm{H} / \mathrm{B}} \times \vec{v}_{\mathrm{H}}+\left(I_{/ \mathrm{F}}+I_{/ \mathrm{K}}+I_{\mathrm{H}}\right) \vec{\Omega}^{-}=M_{s} \vec{r}_{\mathrm{F} / \mathrm{B}} \times \vec{v}_{\mathrm{F}}^{+}+I_{/ \mathrm{F}} \vec{\Omega}_{\mathrm{F}}^{+}+ \\
& M_{t} \vec{r}_{\mathrm{K} / \mathrm{B}} \times \vec{v}_{\mathrm{K}}^{+}+I_{/ \mathrm{K}} \vec{\Omega}_{\mathrm{K}}^{+}+ \\
& M \vec{r}_{\mathrm{H} / \mathrm{B}} \times \vec{v}_{\mathrm{H}}^{+}+I_{/ \mathrm{H}} \vec{\Omega}_{\mathrm{H}}^{+}, \\
& M_{t} \vec{r}_{\mathrm{K} / \mathrm{C}} \times \vec{v}_{\mathrm{K}}^{-}+M \vec{r}_{\mathrm{H} / \mathrm{C}} \times \vec{v}_{\mathrm{H}}+ \\
& \left(I_{/ \mathrm{K}}+I_{/ \mathrm{H}}\right) \vec{\Omega}^{-}=M_{t} \vec{r}_{\mathrm{K} / \mathrm{C}} \times \vec{v}_{\mathrm{K}}^{+}+I_{/ \mathrm{K}} \vec{\Omega}_{\mathrm{K}}^{+}+ \\
& M \vec{r}_{\mathrm{H} / \mathrm{C}} \times \vec{v}_{\mathrm{H}}^{+}+I_{/ \mathrm{H}} \vec{\Omega}_{\mathrm{H}}^{+}, \\
& M \vec{r}_{H / D} \times \vec{v}_{\mathrm{H}}+I_{/ \mathrm{H}} \vec{\Omega}^{-}=M \vec{r}_{\mathrm{H} / \mathrm{D}} \times \vec{v}_{\mathrm{H}}^{+}+I_{/ \mathrm{H}} \vec{\Omega}_{\mathrm{H}}^{+}
\end{aligned}
$$


where $I_{/ \mathrm{E}}, I_{/ \mathrm{F}}, I_{/ \mathrm{K}}, I_{/ \mathrm{H}}$ are moments of inertia of the foot, shank, thigh, and torso segments, respectively about their centers. The linear and angular velocities of the foot $\left(\vec{v}_{\mathrm{E}}, \vec{\Omega}\right)$, shank $\left(\vec{v}_{\mathrm{F}}, \vec{\Omega}_{\mathrm{F}}\right)$, thigh $\left(\vec{v}_{\mathrm{K}}, \vec{\Omega}_{\mathrm{K}}\right)$, and torso $\left(\vec{v}_{\mathrm{H}}, \vec{\Omega}_{\mathrm{H}}\right)$ are related to the velocity of the contact point $\mathrm{O}$ as,

$$
\begin{aligned}
\vec{v}_{\mathrm{E}} & =\vec{v}_{\mathrm{O}}+\vec{\Omega}_{\mathrm{E}} \times \vec{r}_{\mathrm{E} / \mathrm{O}}, \\
\vec{v}_{\mathrm{F}} & =\vec{v}_{\mathrm{O}}+\vec{\Omega}_{\mathrm{E}} \times \vec{r}_{\mathrm{B} / \mathrm{O}}+\vec{\Omega}_{\mathrm{F}} \times \vec{r}_{\mathrm{F} / \mathrm{B}}, \\
\vec{v}_{\mathrm{K}} & =\vec{v}_{\mathrm{O}}+\vec{\Omega}_{\mathrm{E}} \times \vec{r}_{\mathrm{B} / \mathrm{O}}+\vec{\Omega}_{\mathrm{F}} \times \vec{r}_{\mathrm{C} / \mathrm{B}}+\vec{\Omega}_{\mathrm{K}} \times \vec{r}_{\mathrm{K} / \mathrm{C}}, \\
\vec{v}_{\mathrm{H}} & =\vec{v}_{\mathrm{O}}+\vec{\Omega}_{\mathrm{E}} \times \vec{r}_{\mathrm{B} / \mathrm{O}}+\vec{\Omega}_{\mathrm{F}} \times \vec{r}_{\mathrm{C} / \mathrm{B}}+\vec{\Omega}_{\mathrm{K}} \times \vec{r}_{\mathrm{D} / \mathrm{C}}+\vec{\Omega}_{\mathrm{H}} \times \vec{r}_{\mathrm{H} / \mathrm{D}}, \\
\text { where } \vec{v}_{\mathrm{O}} & =\vec{v}_{\mathrm{G}}+\vec{\Omega}^{-} \times \vec{r}_{\mathrm{O} / \mathrm{G}}, \\
\text { and } \vec{v}_{\mathrm{O}}^{+} & =0 .
\end{aligned}
$$

Simultaneously solving equations (8)-(9) yields the post-collision velocities for each segment of the L-bar. From these, we calculate the normalized fore-aft collision impulse for the compliant model using equation (7).

\subsection{Statistical methods}

\subsubsection{Sample size}

Sample size could refer to the number of subjects or the number of foot steps that were used in the analyses. The number of subjects recruited was informed by typical participant numbers that were used in similar past studies $[9,15,20]$. There is an additional consideration for sufficiency of sample numbers for the foot placement analysis. The steps should densely sample the approximately $10 \mathrm{~m}$ long central region of the track, where the motion capture system was recording from. The 5237 recorded steps (2511 on uneven I, 2726 on uneven II) are sufficient to densely sample the measurement region assuming a heel-sized patch for each step.

\subsubsection{Statistical analysis and reporting}

A measure of central tendency and a measure of variability of the distributions of step width, step length, center of mass speed, forward foot speed at landing, fore-aft impulse, virtual leg length at touchdown, leg angle at touchdown, net metabolic rate, and meander are reported for each trial. For step width measures, the distribution of step widths within a trial deviated from normality and hence we report the median and the interquartile range of the distribution 
for each trial instead of the mean and standard deviation, as is reported for all other variables (supplementary Fig. S1).

We use three different linear mixed models to determine (a) whether gait variables described above (and in supplementary Table S1) vary with terrain type, (b) whether leg angle at touchdown and decelerating fore-aft impulses covary with forward foot speed at touchdown, and (c) whether the foot placement index $p_{i, j}$ (equation (5)) correlates with the median height or the interquartile range of heights within the terrain region at landing. The statistical models are run using the lmerTest package in $\mathrm{R}$ [40]. We use a linear mixed-model fit by restricted maximum likelihood t-tests with Satterthwaite approximations to degrees of freedom. An ANOVA on the first model tests for the effect of the terrain factor, an ANCOVA on the second model tests for the effect of the terrain factor and the covariate forward foot speed, and an ANCOVA on the third model tests whether the probability of landing in a terrain patch $p_{i, j}$ significantly covaries with the height or unevenness of that terrain patch. Post-hoc pairwise comparisons, where relevant, are performed using the emmeans package in RStudio with p-values adjusted according to Tukey's method.

A measure of central tendency or variability within a trial is the dependent variable $y$ for the first linear mixed model. There are 27 observations for the dependent variable $y$ corresponding to each trial (9 subjects running on 3 terrain). Terrain is the fixed factor and subjects are random factors in the model given by,

$$
y_{i j}=\left(\beta_{0}+\mu_{j}\right)+\beta_{i} \operatorname{terrain}_{i}+\epsilon_{i j},
$$

where $i=1,2$ and $j=1 \ldots 9$. The intercept $\beta_{0}$ (value of $y$ on flat terrain) and parameters $\beta_{i}$ for uneven I and uneven II are estimated for this model. The random factor variables $\mu_{j}$ are assumed to be normally distributed about zero and account for inter-subject variability of the intercept. The model residuals are $\epsilon_{i j}$ which are also assumed to be normally distributed about zero. Detailed statistics for these tests are reported in supplementary Table S1.

The second linear mixed model uses stepwise data where each step is grouped by subject and terrain type. Each of the 1086 steps in this dataset contains a value for subject number, terrain type, touchdown leg angle, decelerating fore-aft impulse, and forward foot speed at touchdown. 
bioRxiv preprint doi: https://doi.org/10.1101/2021.02.22.432238; this version posted February 22, 2021. The copyright holder for this preprint (which was not certified by peer review) is the author/funder, who has granted bioRxiv a license to display the preprint in perpetuity. It is made available under aCC-BY 4.0 International license.

The linear model for the dependent variable $y$ (touchdown leg angle or fore-aft impulse) is,

$$
y_{i j}=\left(\beta_{0}+\mu_{1 j}\right)+\beta_{i} \text { terrain }_{i}+\left(\beta_{f}+\mu_{2 j}+\nu_{i}\right) \text { footspeed }+\epsilon_{i j}
$$

where $i=1,2$ and $j=1 \ldots 9$. Like in equation (10), the model estimates the intercept $\beta_{0}$, i.e. the value of $y$ on flat terrain when foot speed $=0, \beta_{i}$ for terrain factor, and the slope $\beta_{f}$ for the dependence of $y$ on forward foot speed at touchdown. The variable $\mu_{1 j}$ account for inter-subject variability of the intercept, and the variables $\mu_{2 j}$ and $\nu_{i}$ account for inter-subject and terrain-specific variability of the slope $\beta_{f}$, respectively. The residuals $\epsilon_{i j}$ are assumed to be normally distributed. We report detailed statistics for this model in supplementary Table S2.

Using a dataset of 5237 steps from all subjects on uneven I and uneven II, we extract landing probabilities for 1628 terrain patches (as detailed in section 1.2.2). To test whether runners aimed for terrain regions with low unevenness, we use a linear mixed model of the form,

$$
y_{k l}=\left(\mu_{1 l}+\nu_{1 k}\right)+\left(\mu_{2 l}+\nu_{1 k}\right) \text { terr }+\epsilon_{k l}
$$

where $k=1,2$ for the two uneven terrain and $l=1 \rightarrow 9$ for the 9 subjects. The dependent variable $y$ is the probability of landing in a foot-sized cell $p_{i, j}$ and the independent variable 'terr' refers to the median terrain height of the cell or the interquartile range of heights within the cell. The variables $\mu_{1 l}$ accounts for subject-specific variability in the terrain-specific intercept $\nu_{1 k}$. The variables $\mu_{2 l}$ accounts for subject-specific variability in the terrain-specific slope $\nu_{2 k}$. ANCOVAs on the model for both independent variables (the median terrain height and the interquartile range of heights within the cell) are summarized in Table 1.

Data availability statement: All data points are plotted in either the main text or the electronic supplementary material. Raw data are available on the Dryad repository associated with this paper.

Author Contributions: MV conceived the study. ND conducted the experiment. ND and MV performed the data analysis and wrote the paper.

Acknowledgements: Human Frontier Science Program and Wellcome Trust-DBT Alliance for funding.

Author Declaration: That authors declare that they have no conflict of interest. 
bioRxiv preprint doi: https://doi.org/10.1101/2021.02.22.432238; this version posted February 22, 2021. The copyright holder for this preprint (which was not certified by peer review) is the author/funder, who has granted bioRxiv a license to display the preprint in perpetuity. It is made available under aCC-BY 4.0 International license.

\section{References}

[1] Carrier, D. R. The energetic paradox of human running and hominid evolution. Current Anthropology 25 (1984).

[2] Bramble, D. M. \& Lieberman, D. E. Endurance running and the evolution of homo. Nature 432, 345-352 (2004).

[3] Lee, D. N. \& Lishman, R. Visual control of locomotion. Scandinavian Journal of Psychology 18, 224-230 (1977).

[4] Warren Jr, W. H., Young, D. S. \& Lee, D. N. Visual control of step length during running over irregular terrain. Journal of Experimental Psychology: Human Perception and Performance 12, 259 (1986).

[5] Matthis, J. S., Yates, J. L. \& Hayhoe, M. M. Gaze and the control of foot placement when walking in natural terrain. Curr Biol 28, 1224-1233.e5 (2018).

[6] Thomas, N. D., Gardiner, J. D., Crompton, R. H. \& Lawson, R. Keep your head down: Maintaining gait stability in challenging conditions. Human Movement Science 73, 102676 (2020).

[7] Pontzer, H. Economy and endurance in human evolution. Current Biology 27, R613-R621 (2017).

[8] Daley, M. A., Usherwood, J. R., Felix, G. \& Biewener, A. A. Running over rough terrain: guinea fowl maintain dynamic stability despite a large unexpected change in substrate height. Journal of Experimental Biology 209, 171-187 (2006).

[9] Voloshina, A. S. \& Ferris, D. P. Biomechanics and energetics of running on uneven terrain. Journal of Experimental Biology 218, 711-719 (2015).

[10] Müller, R., Häufle, D. F. B. \& Blickhan, R. Preparing the leg for ground contact in running: the contribution of feed-forward and visual feedback. Journal of Experimental Biology 218, 451-457 (2015).

[11] Müller, R., Birn-Jeffery, A. \& Blum, Y. Human and avian running on uneven ground: a model-based comparison. Journal of The Royal Society Interface 13, 20160529 (2016).

[12] Heim, S., Millard, M., Le Mouel, C. \& Badri-Spröwitz, A. A little damping goes a long way: a simulation study of how damping influences task-level stability in running. Biology Letters 16, 20200467 (2020).

[13] Seyfarth, A., Geyer, H., Günther, M. \& Blickhan, R. A movement criterion for running. Journal of biomechanics 35, 649-655 (2002).

[14] Birn-Jeffery, A. V. et al. Don’t break a leg: running birds from quail to ostrich prioritise leg safety and economy on uneven terrain. Journal of Experimental Biology 217, 3786-3796 (2014).

[15] Seethapathi, N. \& Srinivasan, M. Step-to-step variations in human running reveal how humans run without falling. eLife 8, e38371 (2019).

[16] Karssen, J. D., Haberland, M., Wisse, M. \& Kim, S. The effects of swing-leg retraction on running performance: analysis, simulation, and experiment. Robotica 33, 2137-2155 (2015).

[17] Mahaki, M., Bruijn, S. \& Van Dieën, J. The effect of external lateral stabilization on the use of foot placement to control mediolateral stability in walking and running. PeerJ 7:e7939 (2019).

[18] Birn-Jeffery, A. V. \& Daley, M. A. Birds achieve high robustness in uneven terrain through active control of landing conditions. Journal of Experimental Biology 215, 2117-2127 (2012).

[19] Dhawale, N., Mandre, S. \& Venkadesan, M. Dynamics and stability of running on rough terrains. Royal Society Open Science 6, 181729 (2019).

[20] Donelan, J. M., Shipman, D. W., Kram, R. \& Kuo, A. D. Mechanical and metabolic requirements for active lateral stabilization in human walking. Journal of biomechanics 37, 827-835 (2004).

[21] Blum, Y., Lipfert, S., Rummel, J. \& Seyfarth, A. Swing leg control in human running. Bioinspiration $\mathcal{E}^{8}$ biomimetics 5, 026006 (2010).

[22] Arellano, C. J. \& Kram, R. The energetic cost of maintaining lateral balance during human running. Journal of Applied Physiology 112, 427-434 (2012). 
[23] Alexander, R. \& Jayes, A. A dynamic similarity hypothesis for the gaits of quadrupedal mammals. Journal of zoology 201, 135-152 (1983).

[24] Lieberman, D. E. et al. Foot strike patterns and collision forces in habitually barefoot versus shod runners. Nature 463, 531 (2010).

[25] Chatterjee, A. \& Ruina, A. Two interpretations of rigidity in rigid-body collisions. Journal of Applied Mechanics 65, 894-900 (1998).

[26] Blum, Y. et al. Swing-leg trajectory of running guinea fowl suggests task-level priority of force regulation rather than disturbance rejection. PloS one 9, e100399 (2014).

[27] Seyfarth, A., Geyer, H. \& Herr, H. Swing-leg retraction: a simple control model for stable running. Journal of Experimental Biology 206, 2547-2555 (2003).

[28] Seipel, J. E. \& Holmes, P. Running in three dimensions: Analysis of a point-mass sprung-leg model. The International Journal of Robotics Research 24, 657-674 (2005).

[29] Blum, Y., Birn-Jeffery, A., Daley, M. A. \& Seyfarth, A. Does a crouched leg posture enhance running stability and robustness? Journal of Theoretical Biology 281, 97-106 (2011).

[30] Matthis, J. S. \& Fajen, B. R. Visual control of foot placement when walking over complex terrain. Journal of experimental psychology: human perception and performance 40, 106 (2014).

[31] Cavanagh, P. R. \& Williams, K. R. The effect of stride length variation on oxygen uptake during distance running. Medicine and science in sports and exercise 14, 30-35 (1981).

[32] Arellano, C. J. \& Kram, R. The effects of step width and arm swing on energetic cost and lateral balance during running. Journal of biomechanics 44, 1291-1295 (2011).

[33] Venkadesan, M., Mandre, S. \& Bandi, M. M. Bioinspired Legged Locomotion: Models, Concepts, Control and Applications, Chapter 7 (Butterworth-Heinemann, 2017).

[34] Donelan, J. M., Kram, R. \& Kuo, A. D. Mechanical and metabolic determinants of the preferred step width in human walking. Proceedings of the Royal Society of London B: Biological Sciences 268, 1985-1992 (2001).

[35] Brockway, J. Derivation of formulae used to calculate energy expenditure in man. Human nutrition. Clinical nutrition 41, 463-471 (1987).

[36] Dempster, W. T. Space requirements of the seated operator, geometrical, kinematic, and mechanical aspects of the body with special reference to the limbs. Tech. Rep., Michigan State Univ East Lansing (1955).

[37] Donelan, J. M., Kram, R. \& Kuo, A. D. Mechanical work for step-to-step transitions is a major determinant of the metabolic cost of human walking. Journal of Experimental Biology 205, 3717-3727 (2002).

[38] Ruina, A., Bertram, J. E. \& Srinivasan, M. A collisional model of the energetic cost of support work qualitatively explains leg sequencing in walking and galloping, pseudo-elastic leg behavior in running and the walk-to-run transition. Journal of theoretical biology 237, 170-192 (2005).

[39] Srinivasan, M. \& Ruina, A. Computer optimization of a minimal biped model discovers walking and running. Nature 439, 72-75 (2006).

[40] Kuznetsova, A., Brockhoff, P. B. \& Christensen, R. H. B. lmerTest package: Tests in linear mixed effects models. Journal of Statistical Software 82, 1-26 (2017). 


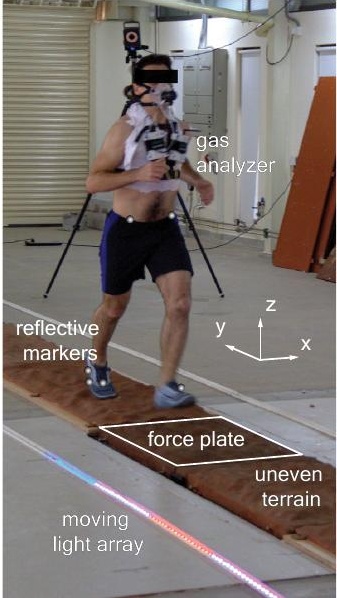

b

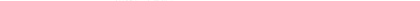




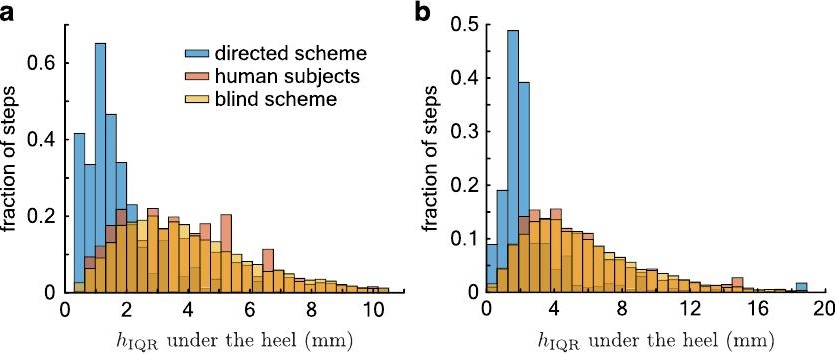


b
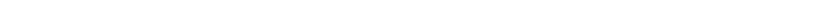

d
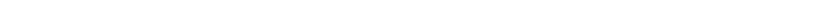


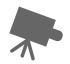

$3 \mathrm{~m}$
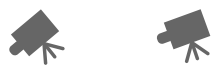

LED strip
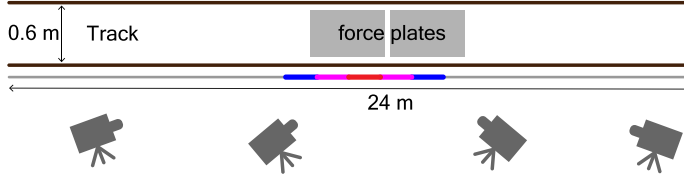

b
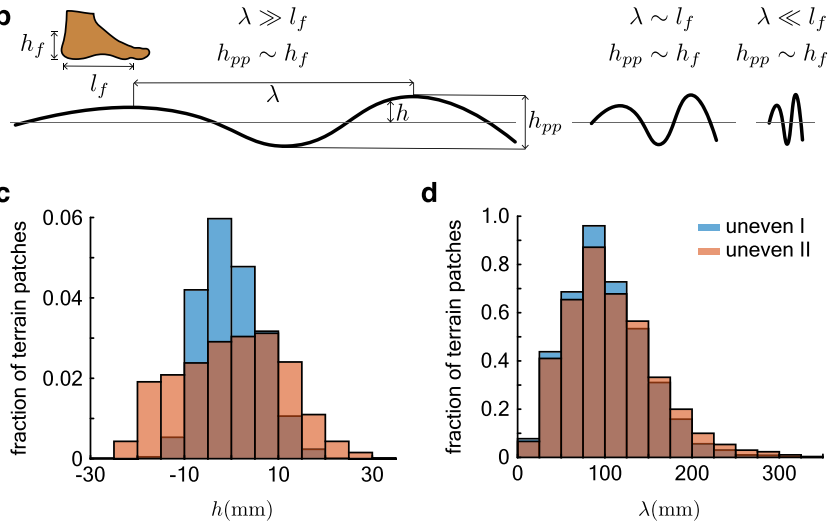


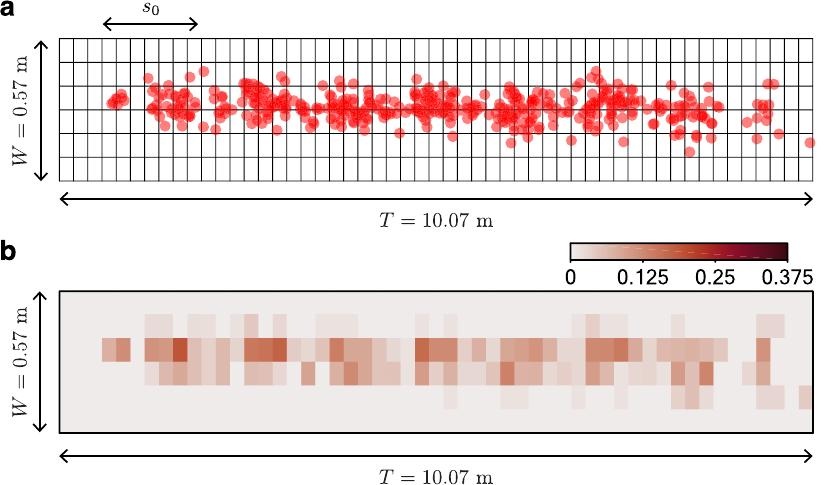




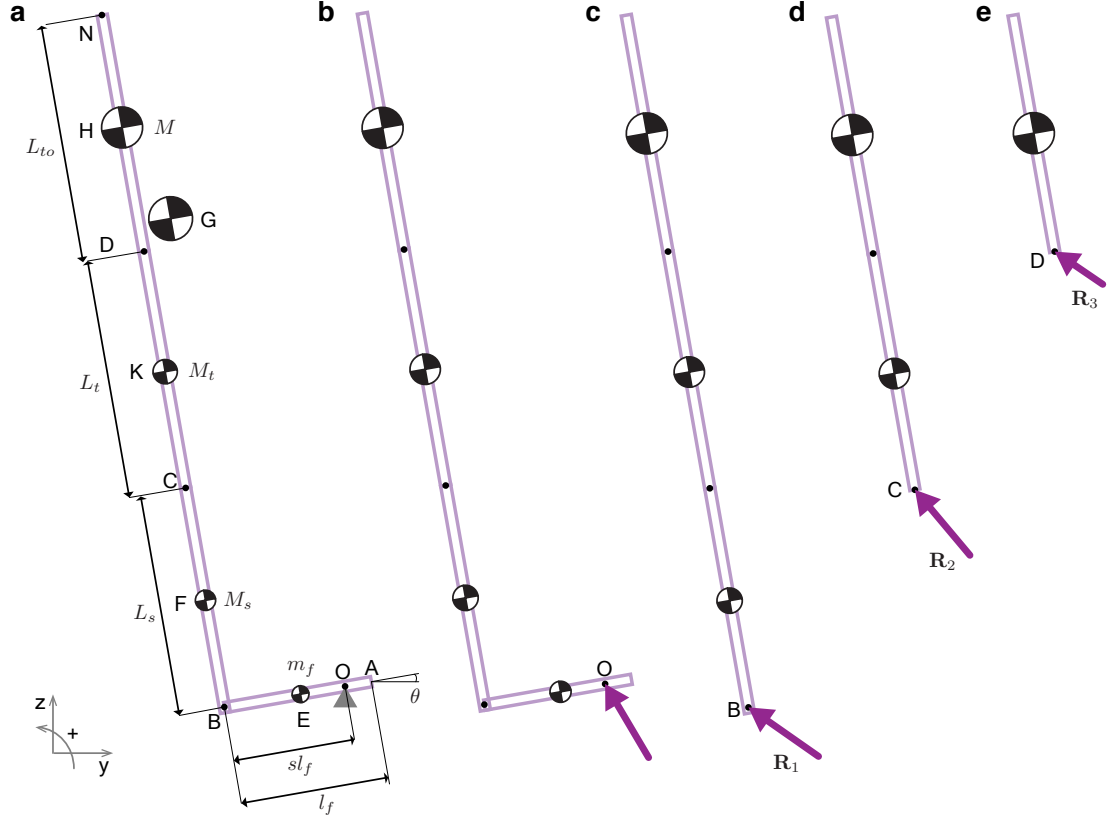




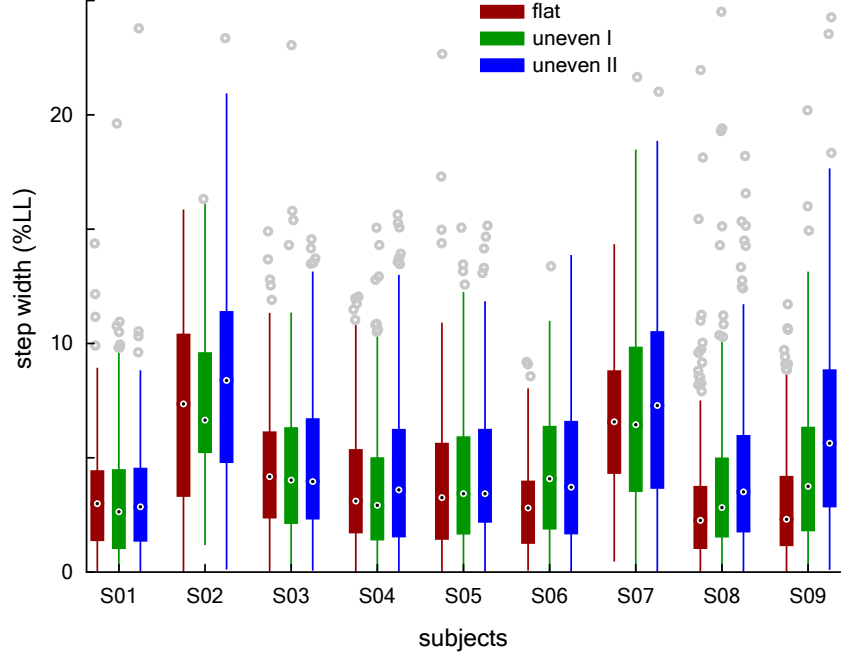

Figure S1. Subject-wise step width statistics. Step width is expressed as percent leg length (LL). Because the data are skewed, we report the median and interquartile range as measures of the central tendency and variability, respectively. Black dots represent medians, boxes show the interquartile range, lines extend to 1.5 times the quartile range, and grey circles represent outliers. 

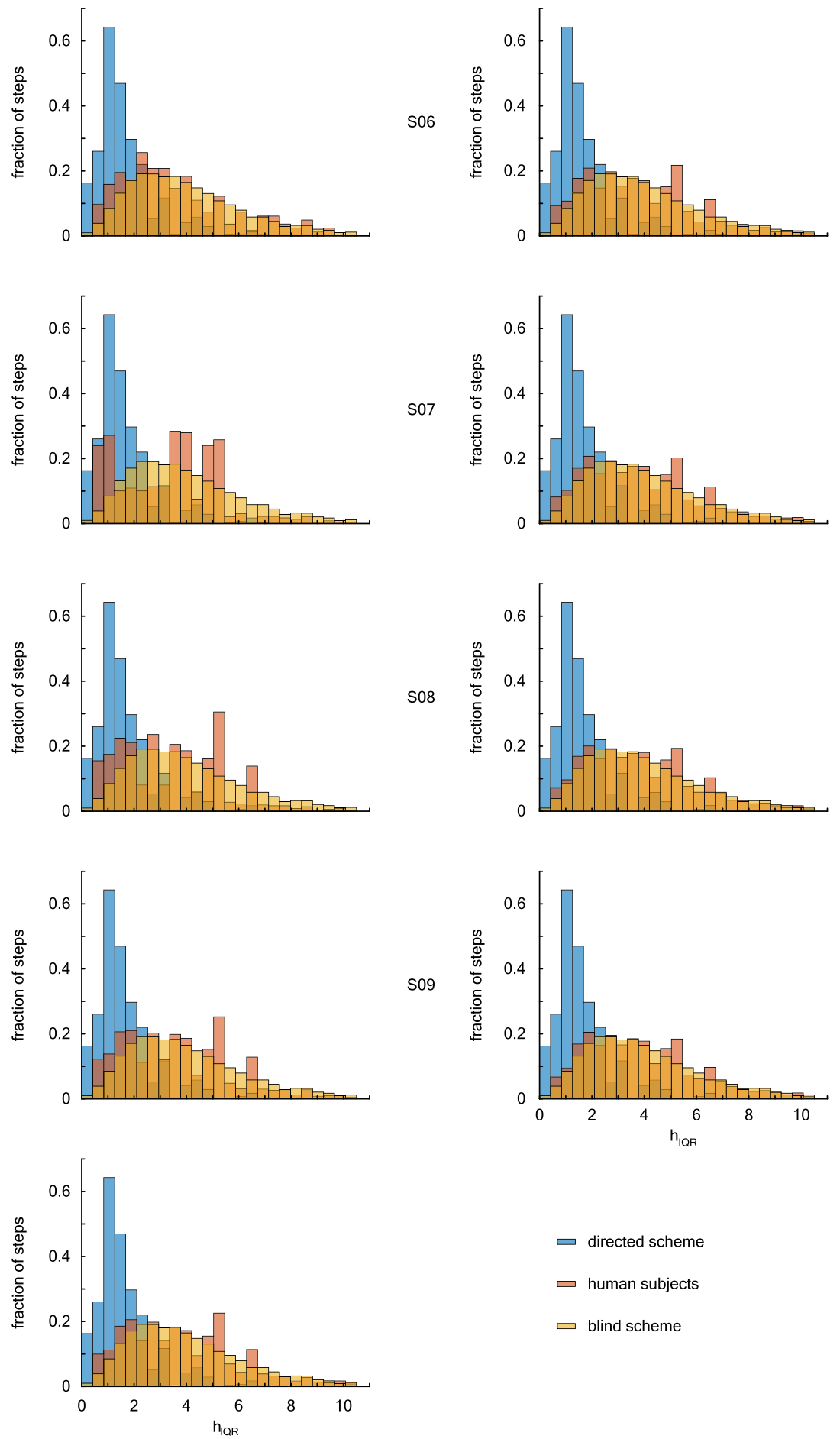

Figure S2. Subject-wise foot placement analysis on uneven I. Histograms of the interquartile range of heights (IQR) at the footstep locations for the directed sampling scheme (blue), each subject from the experiments (red), and the blind sampling scheme (yellow). 

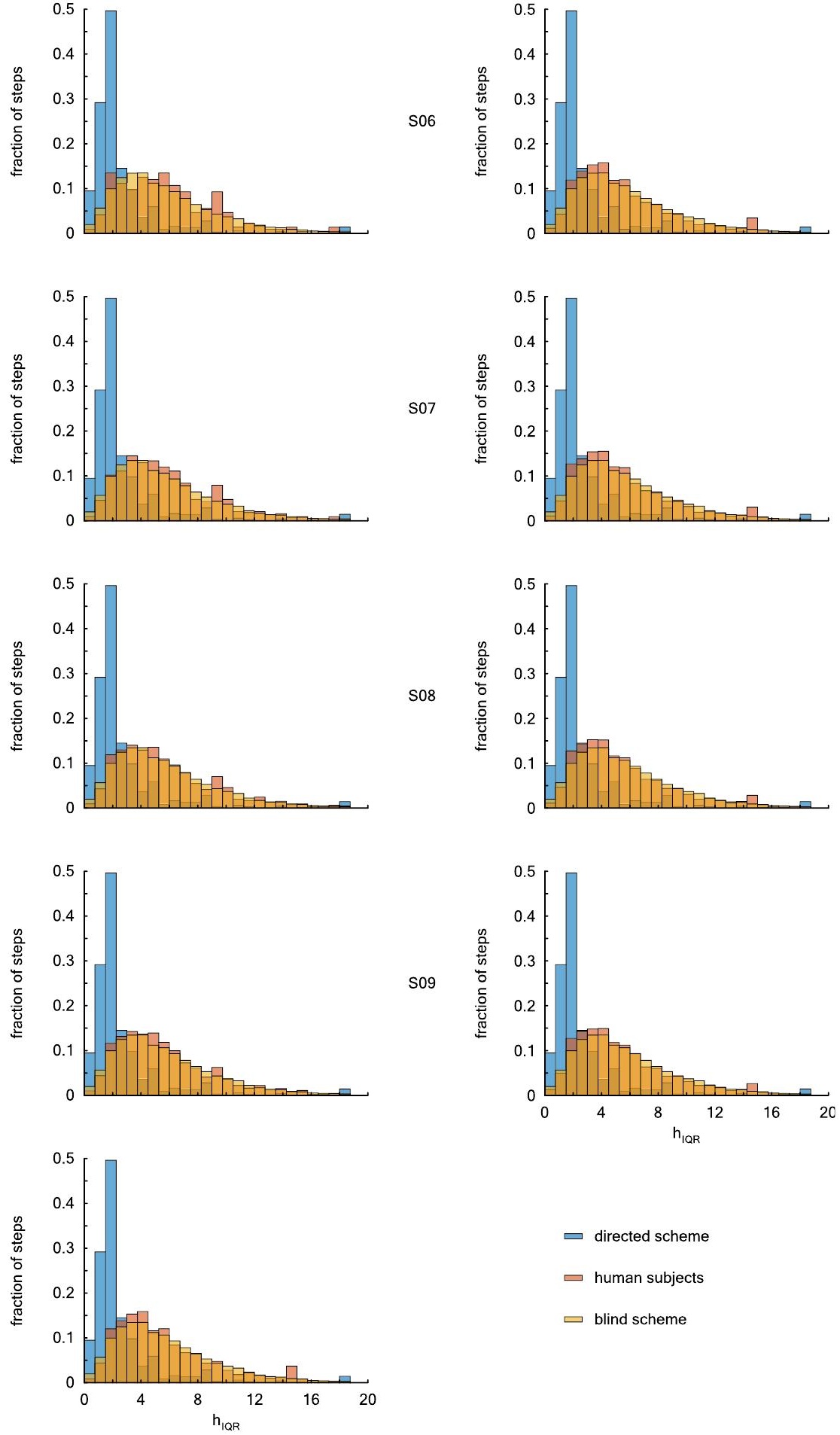

Figure S3. Subject-wise foot placement analysis on uneven II. Histograms of the interquartile range of heights (IQR) at the footstep locations for the directed sampling scheme (blue), each subject from the experiments (red), and the blind sampling scheme (yellow). 
$\varepsilon$
5
0
0

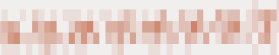

$10.07 \mathrm{~m}$

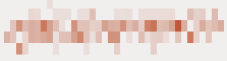

subject 07

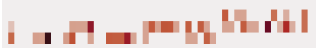

|rhathinghth

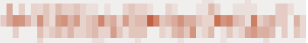

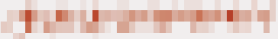

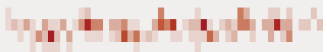

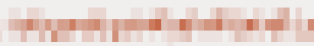

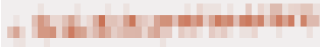

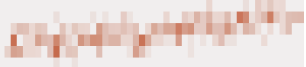

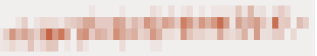

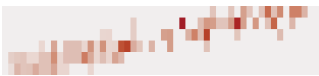

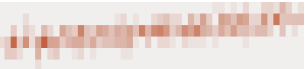

4hingurmunty

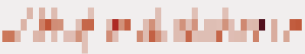

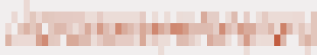

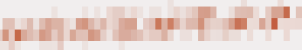

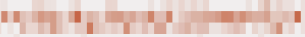

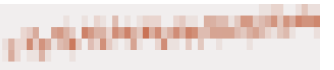

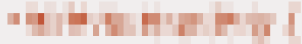

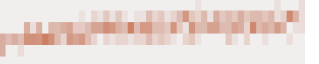

Figure S4. Subject-wise foot placement patterns. Heatmaps of the foot placement index for all subjects on each terrain. Each cell has an area of $190 \mathrm{~mm}$ $\times 95 \mathrm{~mm}$, with the longer side of the rectangle along the length of the track. Colour bar for the heatmaps in the bottom left of the figure. 
subject 01

$=0.2$. 180.80900

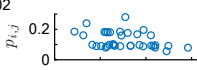

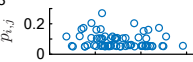

subject 04

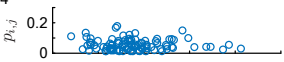

subject 05

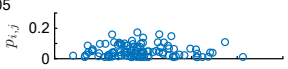

subject 06

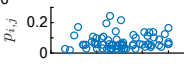

subject 07

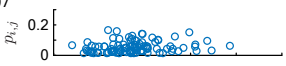
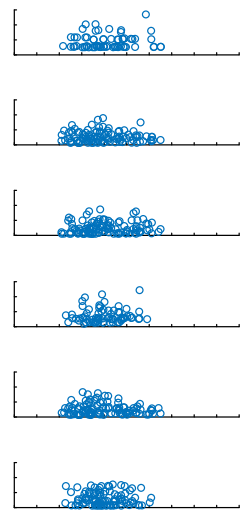

2. 0.2 [ $0.89808,8$.

subject 09

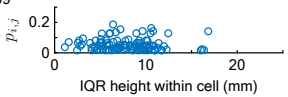

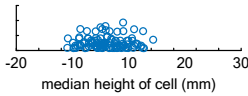

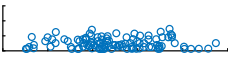

[

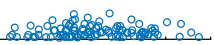

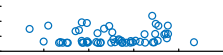

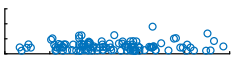

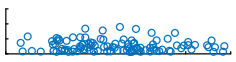

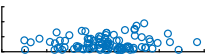

$\circ 000988^{\circ} 0^{\circ} \circ$.

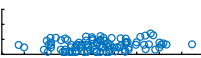

[

$\left[\begin{array}{r}0 \\ 0.0008 \\ 0\end{array}\right.$
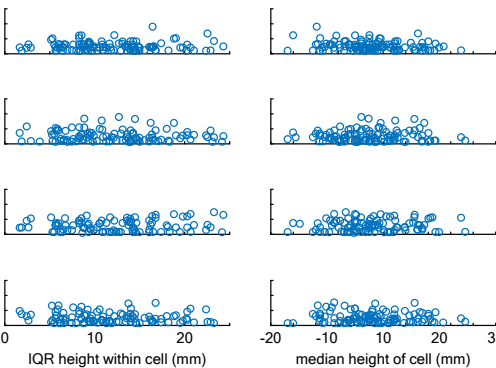

Figure S5. Subject-wise foot placement analysis. Foot placement index $p_{i, j}$ plotted against the median height of the terrain cell and the interquartile range of heights within the terrain cell at landing for all recorded steps on uneven I and uneven II. 
Table S1. Kinematic variables on different terrain types reported as mean \pm $\mathrm{SD}$, except for meander values which are reported as median \pm interquartile range. For each variable, we show details of the ANOVAs performed on the linear model (from main text equation (10)), i.e. the F-value and p-value for the terrain factor. The denominator degrees of freedom for all ANOVAs was 16. Post-hoc comparisons when the ANOVAs reached the significance bound of $\alpha=0.05$ are reported in the main text results section.

\begin{tabular}{|c|c|c|c|c|c|}
\hline variable & flat & uneven I & uneven II & F-value & p-value \\
\hline net metabolic rate $(\mathrm{W} / \mathrm{kg})$ & $13.1 \pm 0.5$ & $13.7 \pm 0.9$ & $13.7 \pm 0.8$ & 2.97 & 0.08 \\
\hline median step width (\%LL) & $3.9 \pm 1.9$ & $4.1 \pm 1.5$ & $4.7 \pm 2.0$ & 4.53 & 0.03 \\
\hline IQR step width (\% LL) & $3.9 \pm 1.4$ & $4.3 \pm 0.9$ & $5.0 \pm 1.2$ & 3.65 & 0.05 \\
\hline mean step width (\%LL) & $4.2 \pm 1.7$ & $4.7 \pm 1.6$ & $5.2 \pm 1.7$ & 8.69 & 0.003 \\
\hline S.D. step width (\% LL) & $2.8 \pm 0.8$ & $3.4 \pm 0.6$ & $3.6 \pm 0.6$ & 5.54 & 0.01 \\
\hline mean step length (\%LL) & $128 \pm 6$ & $126 \pm 9$ & $125 \pm 9$ & 1.07 & 0.37 \\
\hline S.D. step length (\%LL) & $6 \pm 1$ & $7 \pm 4$ & $6 \pm 1$ & 0.64 & 0.54 \\
\hline mean meander $\left(\times 10^{-4}\right)$ & $3.21 \pm 2.59$ & $3.97 \pm 1.65$ & $4.88 \pm 4.62$ & 1.48 & 0.25 \\
\hline S.D. meander $\left(\times 10^{-4}\right)$ & $0.67 \pm 0.53$ & $1.33 \pm 1.40$ & $1.27 \pm 2.78$ & 1.58 & 0.23 \\
\hline mean fwd. foot speed (froude num.) & $0.53 \pm 0.17$ & $0.36 \pm 0.10$ & $0.37 \pm 0.12$ & 13.08 & 0.0004 \\
\hline S.D. fwd. foot speed (froude num.) & $0.17 \pm 0.05$ & $0.14 \pm 0.05$ & $0.18 \pm 0.07$ & 1.48 & 0.26 \\
\hline mean CoM speed $(\mathrm{m} / \mathrm{s})$ & $3.24 \pm 0.07$ & $3.21 \pm 0.07$ & $3.18 \pm 0.09$ & 2.32 & 0.13 \\
\hline S.D. CoM speed $(\mathrm{m} / \mathrm{s})$ & $0.11 \pm 0.03$ & $0.13 \pm 0.04$ & $0.12 \pm 0.03$ & 2.00 & 0.17 \\
\hline mean touchdown leg length (\%LL) & $120 \pm 5$ & $119 \pm 4$ & $119 \pm 4$ & 4.28 & 0.03 \\
\hline S.D. touchdown leg length (\%LL) & $1.1 \pm 0.7$ & $0.9 \pm 0.3$ & $1.3 \pm 1.2$ & 1.32 & 0.29 \\
\hline mean touchdown leg angle (rad) & $0.20 \pm 0.02$ & $0.20 \pm 0.02$ & $0.21 \pm 0.02$ & 3.90 & 0.04 \\
\hline S.D. touchdown leg angle (rad) & $0.03 \pm 0.02$ & $0.02 \pm 0.003$ & $0.03 \pm 0.02$ & 2.10 & 0.15 \\
\hline
\end{tabular}


Table S2. Details of the ANCOVAs performed on the linear model (from main text equation (11)) showing the denominator degrees of freedom, F-value and p-value for the fixed terrain factor, and the estimated slopes $\beta_{f}$ for the fixed forward foot speed effect.

\begin{tabular}{llllll}
\hline dependent variable & factor & DenDF & F-value & p-value & $\beta_{f}$ \\
\hline touchdown leg angle & terrain & 193 & 1.48 & 0.23 & - \\
& fwd. foot speed & 38 & 115.83 & $<0.0001$ & 0.07 \\
fore-aft impulse & terrain & 79 & 1.45 & 0.24 & - \\
& fwd. foot speed & 78 & 12.83 & 0.001 & 0.01 \\
\hline
\end{tabular}


Table S3. Footstep counts for each subject on all terrain.

\begin{tabular}{cccc}
\hline subject & flat & uneven I & uneven II \\
\hline 1 & 363 & 321 & 448 \\
2 & 228 & 78 & 473 \\
3 & 473 & 131 & 436 \\
4 & 373 & 471 & 519 \\
5 & 366 & 557 & 109 \\
6 & 224 & 160 & 327 \\
7 & 218 & 398 & 442 \\
8 & 503 & 489 & 479 \\
9 & 477 & 390 & 392
\end{tabular}

